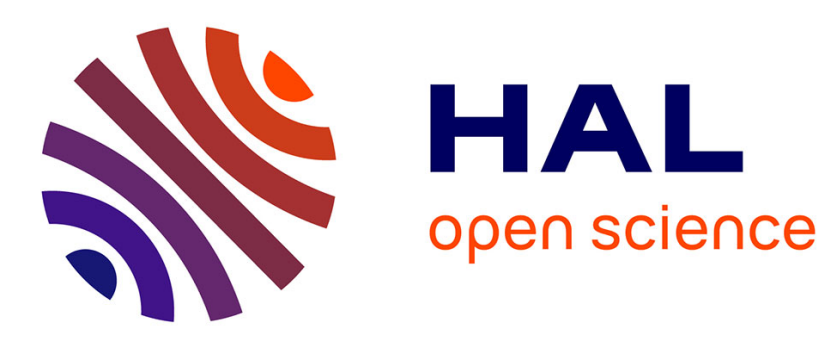

\title{
Bioinspired Photoredox Benzylation of Quinones
}

\author{
Maxime Donzel, Mourad Elhabiri, Elisabeth Davioud-Charvet
}

\section{To cite this version:}

Maxime Donzel, Mourad Elhabiri, Elisabeth Davioud-Charvet. Bioinspired Photoredox Benzylation of Quinones. Journal of Organic Chemistry, 2021, 86 (15), pp.10055-10066. 10.1021/acs.joc.1c00814 . hal-03369963

\section{HAL Id: hal-03369963 \\ https://hal.science/hal-03369963}

Submitted on 7 Oct 2021

HAL is a multi-disciplinary open access archive for the deposit and dissemination of scientific research documents, whether they are published or not. The documents may come from teaching and research institutions in France or abroad, or from public or private research centers.
L'archive ouverte pluridisciplinaire HAL, est destinée au dépôt et à la diffusion de documents scientifiques de niveau recherche, publiés ou non, émanant des établissements d'enseignement et de recherche français ou étrangers, des laboratoires publics ou privés. 


\title{
A Bioinspired Photoredox Benzylation of Quinones
}

\author{
Maxime Donzel, Mourad Elhabiri, Elisabeth Davioud-Charvet* \\ Université de Strasbourg-CNRS-UHA UMR7042, Laboratoire d'Innovation Moléculaire et Applications (LIMA) Team \\ Bio(IN)organic and Medicinal Chemistry, European School of Chemistry, Polymers and Materials (ECPM), 25 Rue Becque- \\ rel, Strasbourg 67087, France
}

Supporting Information Placeholder

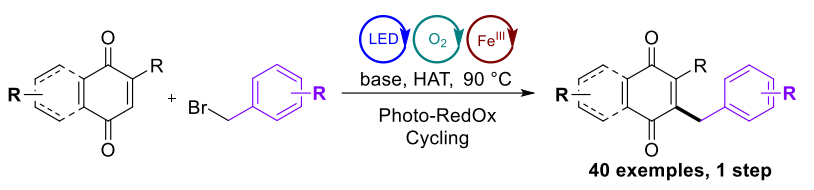

ABSTRACT: 3-Benzylmenadiones were obtained in good yields by using a blue light-induced photoredox process in the presence of Fe(III), oxygen and $\gamma$-terpinene acting as a HAT agent. This methodology is compatible with a wide variety of diversely substituted 1,4-naphthoquinones as well as various cheap, readily available benzyl bromides with excellent functional group tolerance. The benzylation mechanism was investigated and supports a three-step radical cascade with the key involvement of photogenerated superoxide anion radical.

\section{INTRODUCTION}

Quinones have recently shown a re-gaining interest in medicinal chemistry, particularly in the field of drug discovery for new treatments against neglected tropical parasitic diseases such as malaria. In particular, an early lead 3-benzylmenadione named plasmodione (i.e., 3-[4-(trifluoromethyl)benzyl]-menadione) has shown potent and valuable antiplasmodial activities against several Plasmodium falciparum strains in vitro and in Plasmodium berghei-infected mouse model. ${ }^{l}$ In recent years, our team has developed new synthetic methodologies ${ }^{2 a b}$ to introduce structural and functional diversity for reaching more active ${ }^{2 c}$ and water-soluble ${ }^{2 d}$ antiparasitic analogues, or to prepare putative metabolites ${ }^{2 b e}$ to investigate the mechanism of action of these drug candidates ${ }^{l b, 2 f}$. Based on a Kochi-Anderson radical decarboxylation of $\operatorname{acids}^{3 a-c}$, the former extensively investigated synthetic route to build 3-benzylmenadiones suffered from the use of silver salts and peroxodisulfate and was limited by the availability of phenylacetic acids used as starting materials. $^{l a, 3 d, e}$ Since, Lee's group developed an elegant silver-free alkylation of quinones (Scheme 1a) ${ }^{4}$ and a growing interest in benzylated quinones, including plasmodione, has emerged, as evidenced by the synthetic procedures recently described by several groups from substituted toluenes by free-radical generation via hydrogen-atom abstraction using the selectfluor/Ag(I) pair ${ }^{5 a}$ or peroxides (Scheme $1 b)^{5 b, c}$. Finally, when carrying out the work described here, Li et al. concomitantly demonstrated that benzyl radicals could be generated at high temperature in the presence of iron(II) and a base to further react with quinones or coumarins $(\text { Scheme 1c })^{5 d}$.

Our initial goal was to overcome the limitations and drawbacks of silver-catalyzed radical benzylation. To increase the chemical space available around this promising scaffold of benzylated quinones, it appeared essential to develop a simple, robust and efficient strategy to generate, by an eco-friendly process, benzyl radicals from cheap and easily available precursors such as benzyl bromides. The latter are common building blocks in organic chemistry, widely available and known for their ability to generate after reduction of the $\mathrm{C}-\mathrm{Br}$ bond benzyl radical intermediates ${ }^{5 d, 6}$, especially in the emerging field of photoredox catalysis. ${ }^{7}$

Scheme 1. Reported benzylation reactions of quinones and this work.

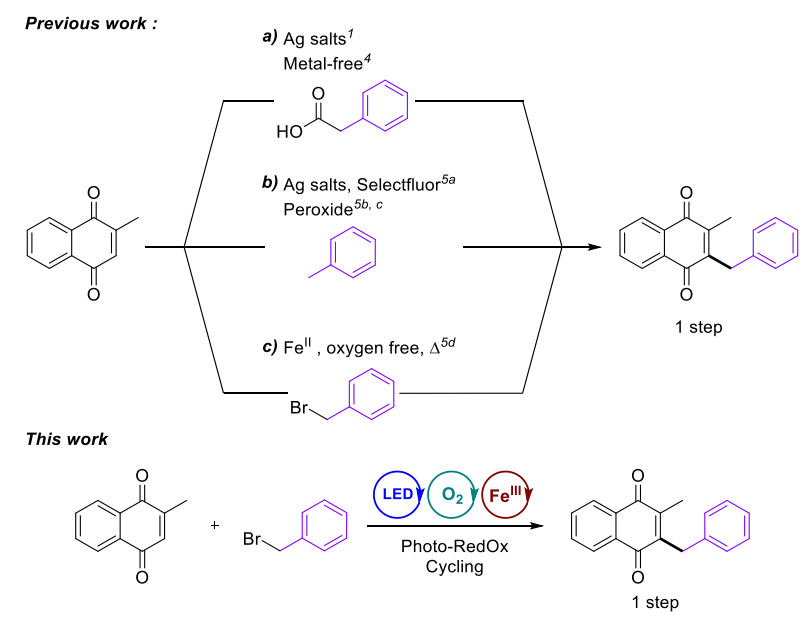

\section{RESULTS AND DISCUSSION}

To this purpose, we first investigated classical benzyl radical generation via transition metal catalyzed photoredox process. ${ }^{7 c, 8}$ However, all attempts failed because these reactions take place under reducing conditions, which are not compatible with the requirement of keeping menadione under its oxidized state. Then, we considered the original work described by Melchiorre and his team. ${ }^{9}$ In this report, after an initial nucleophilic substitution on the electrophilic $\mathrm{C}-\mathrm{Br}$ position of a benzyl bromide, the newly formed $\mathrm{C}-\mathrm{S}$ bond was cleaved under blue light irradiation and mild heating to generate a 
benzyl radical, which can readily react with a radical acceptor. These conditions were first applied to menadione 1a acting as a radical acceptor and 4-trifluoromethylbenzyl bromide $\mathbf{2 a}$ to form the targeted model plasmodione 3a. Detailed NMR analysis of the crude mixture showed the formation of 3a with a low yield (5\%), together with the starting material under its oxidized (1) and reduced (hydroquinone) states (Table 1, Entry 1). Despite this low yield, this encouraged us to further optimize the experimental conditions. With the purpose to limit hydroquinone formation, we were inspired by the putative mechanism of action of plasmodione in $P$. falciparum-parasitized red blood cells (Figure 1), whereby reduced naphthoquinone is continuously re-oxidized by methemoglobin (FeIII) $\left.\left(\operatorname{metHb}\left(\mathrm{Fe}^{\mathrm{III}}\right)\right)\right)$ at the expense of NADPH. ${ }^{l a},{ }^{2 f}$ Consequently, we introduced an iron(III) source to constantly catalyze the in situ re-oxidation of the hydroquinone into the quinone species 1a. After optimization, the target 3a plasmodione was successfully obtained with $79 \%$ isolated yield (Table 1, Entry 2).

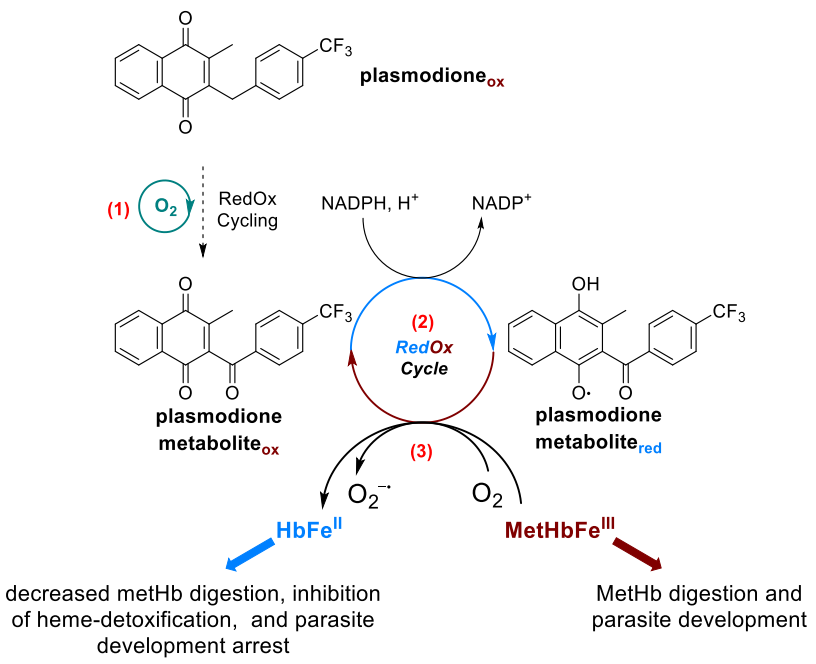

Figure 1. Bioactivation of plasmodione upon internalization in the parasite: plasmodione is proposed to enter into an oxidoreductasemediated redox-cycle in the course of which it is metabolized in its key 3-benzoylmenadione metabolite (step 1). Under its oxidized form, plasmodione metabolite is reduced (step 2), while under its reduced form, it takes part in oxidoreductase-mediated redox-cycling (step 3) leading to decreased methemoglobin (metHb) digestion, inhibition of heme-detoxification, and ROS-induced parasite death.

Several conditions were modified to study their effects and to optimize the parameters of the reaction. We first demonstrated that the nucleophilic organic catalyst (catalyst 1) was not necessary for the reaction to proceed (Table 1 , entry 3 ). In its absence, the target compound 3a was still obtained with an excellent yield of $73 \%$ (versus $81 \%$ ). Afterwards, the concentration was increased up to $0.1 \mathrm{M}$ to achieve a very satisfactory yield of $84 \%$ (Table 1, Entry 4). The iron(III) source can be substituted by any other ferric source such as $\mathrm{Fe}$ (III) chloride or Fe(III) perchlorate and even Fe(II) chloride with, however, slightly lower yields (Table 1, Entry 5-7). For chloride Fe(II), partial oxidation into iron(III) species in solution likely occurs during the reaction. On the other hand, Hantzsch ester (HE) also appears to be a suitable hydrogen-atom transfer (HAT) agent (Table 1, Entry 8). Finally, ambient oxygen was found to play a critical role since nearly no targeted product $\mathbf{3 a}$ was obtained under strict oxygen-free conditions (Table 1, Entry 9). Absence of catalyst, light, $\gamma$-terpinene or base led to very low yields between 0 and $20 \%$ of conversion (See Supporting Information for experimental details). Finally, benzyl iodide 2a' was also shown to react with menadione 1a with a comparable yield of 79\%, as much as benzyl chloride $\mathbf{2 a}$ ", though with a lower yield, thus widening the possibilities in many synthetic applications (Table 1, Entry 10-11).

Table 1. Optimization the experimental conditions of the benzylation reaction of menadione $\mathbf{1} \mathbf{a}^{a}$

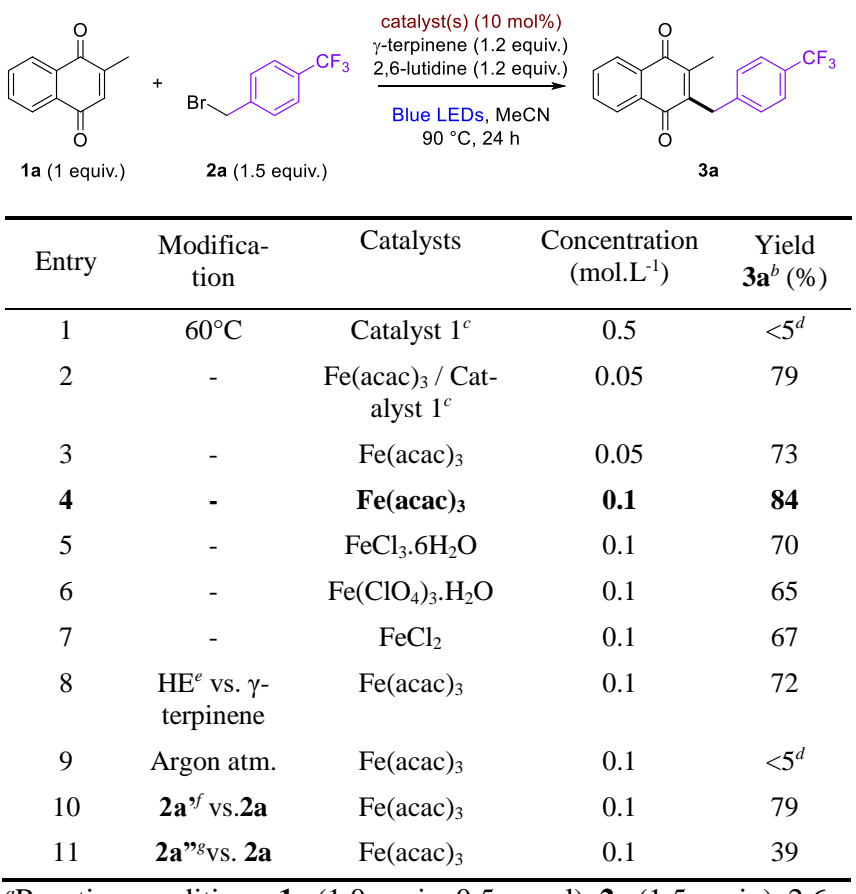

${ }^{a}$ Reaction conditions: 1a (1.0 equiv, $\left.0.5 \mathrm{mmol}\right), 2 \mathrm{a}$ (1.5 equiv), 2.6lutidine (1.2 equiv.), $\gamma$-terpinene (1.2 equiv.), Cat. in $\mathrm{CH}_{3} \mathrm{CN}$ at $90^{\circ} \mathrm{C}$ under blue light irradiation during $24 \mathrm{~h}$. ${ }^{b}$ Isolated yield ${ }^{c}$ Catalyst 1 = potassium 5 -bromo-1H-indole-1-carbodithioate. ${ }^{d}$ Determined by ${ }^{1} \mathrm{H}$ NMR. ${ }^{e} \mathrm{HE}=$ Hantzsch ester $=$ Diethyl 2,6-dimethyl-1,4-dihydropyridine-3,5-dicarboxylate ${ }^{f} \mathbf{2} \mathbf{a}=$ 4-trifluoromethylbenzyl iodide. ${ }^{82 a "}=4$-trifluoromethylbenzyl chloride

Following this optimization stage, we then investigated the scope of this reaction (Scheme 2). First, we demonstrated that the trifluoromethyl substitution was well tolerated both in the meta, ortho or para positions $(76-84 \%, \mathbf{3 a}, \mathbf{3 l}, \mathbf{3 m})$. Plasmodione 3a was also produced in 1 gram scale with good yield demonstrating that this reaction can be scaled up to larger quantities. Various and numerous substitutions have been shown to be compatible with this reaction leading to moderate to good yields in para position (3a-3k). Mono $(\mathbf{3 m}-\mathbf{0}, 3 \mathbf{u}, \mathbf{3 w}, \mathbf{3 x}, \mathbf{3 y})$ or di-substitution (3t) in the ortho-position (steric interactions) does not seem to be a major drawback. On the other hand, a wide variety of poly-substituted benzyl radicals have been also successfully incorporated into menadione (3p-3ab). Finally, we observed that allyl bromide could also be a suitable substrate of this reaction, affording the allylated menadione 3ac with satisfying yield $(68 \%)$ under the same experimental conditions. Then, we evaluated its compatibility with the varied substitution pattern of the quinone unit 4 (Scheme 3). 2-Substitution in 1,4naphthoquinone was found to be well tolerated with moderate to good yields (5a-g). Substitution on the aromatic naphthoquinone ring was also found to be compatible with this reaction, as demonstrated by the successful preparation of the benzyl derivatives with moderate to good yields when starting from plumbagin $(\mathbf{5} \mathbf{h})$, its methyl derivative $(\mathbf{5 i})$ or the 6-fluoro-menadione $(\mathbf{5 j})$. 
Scheme 2. Scope of benzyl bromides with optimized conditions (Table 1, entry 3 )

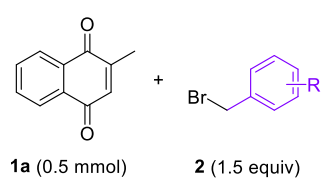

$\mathrm{Fe}(\mathrm{acac})_{3}(10 \mathrm{~mol} \%)$

2,6 lutidine (1.2 equiv.)

Blue LEDs, MeCN $(0.1 \mathrm{M})$ $90^{\circ} \mathrm{C}, 24 \mathrm{~h}$
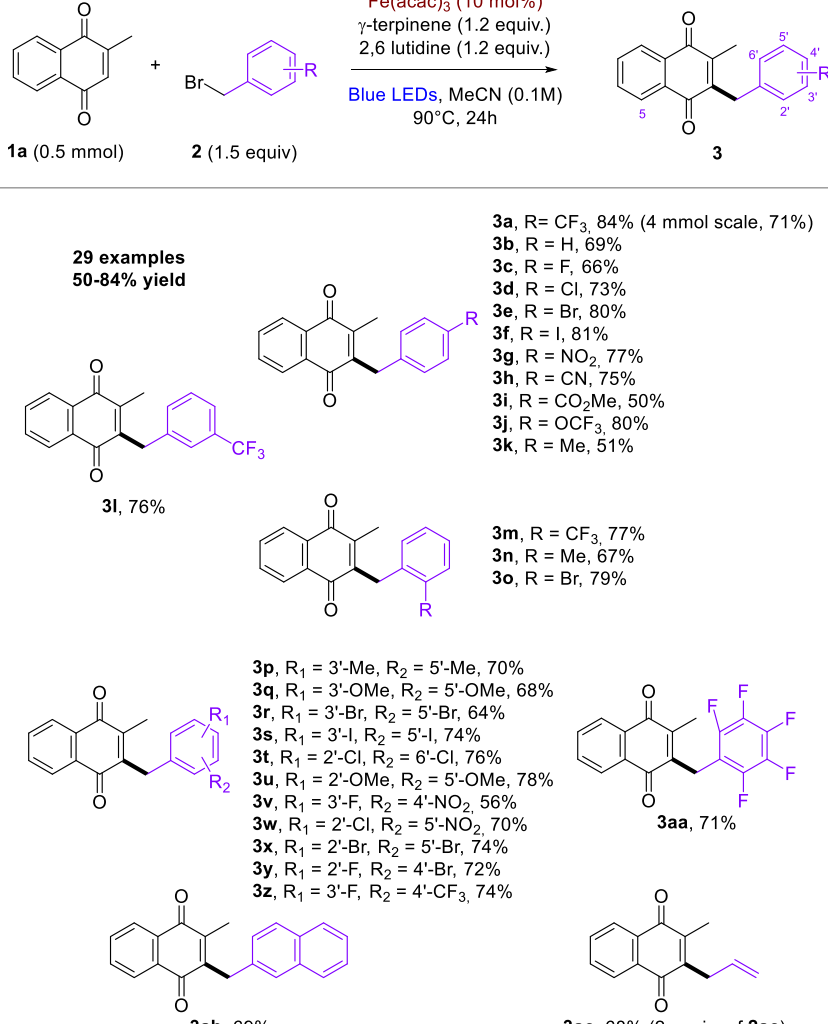

3ab, $69 \%$

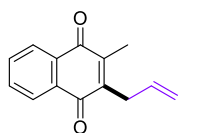

3ac, $68 \%$ ( 2 equiv. of $\mathbf{2 a c}$ )

Scheme 3. Scope of 1,4-quinones with optimized conditions (Table 1 , entry 3 )

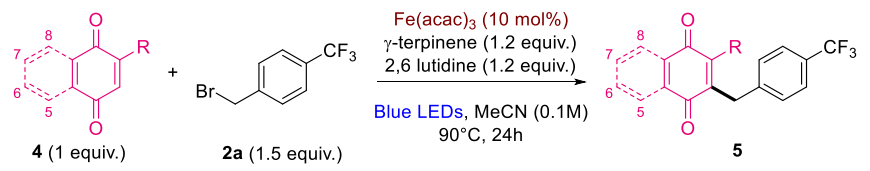

4 (1 equiv.) $\quad 2 a(1.5$ equiv. $)$

5a, $\mathrm{R}=\mathrm{Cl}, 54 \%$

5b, $\mathrm{R}=\mathrm{Br}, 43 \%$

$5 c, R=P h, 89 \%$

5d, $R=E t, 63 \%$

$\mathbf{5 e}, \mathrm{R}=\mathrm{Bn}, 56 \%$
$\mathbf{5 f}, \mathrm{R}=\mathrm{OPh}, 42 \%$

11 examples
$37-89 \%$ yield

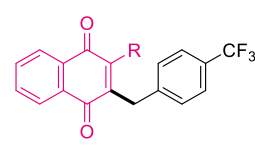

$5 g, R=O A, 49 \%$
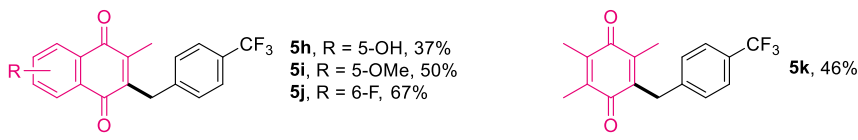

To get a deeper insight into the understanding of the mechanism of the reaction, absorption spectrophotometry and several control experiments were carried out with the model reaction involving 1a and 2a (See Schemes S1-S3 in the Supporting Information). The radical nature of the reaction was assessed by trapping the corresponding radical intermediates, using several radical scavengers (Scheme 4A, and Scheme S1 in the Supporting Information). The reaction was completely inhibited with TEMPO since no trace of the desired 3-benzylmenadione was detected. In addition, complete degradation of the starting menadione was observed. More interestingly, reaction with 1,1-diphenylethylene as radical scavenger afforded the targeted 3-benzylmenadione with a much lower yield together with the trapped benzyl radical adduct. These data support the fact that benzyl radicals are most likely the key intermediates involved in the reaction mechanism. In the process described by Melchiorre and his coworkers ${ }^{9}$, the reactive radical species was generated by a homolytic cleavage of the C-S bond, which was formed after the initial nucleophilic substitution between catalyst 1 and the benzyl bromide. In the absence of this catalyst, the successful conversion to the targeted product suggests that a completely different pathway takes place during the photoreaction.

Scheme 4. Selected mechanistic studies.

A. Radical trap experiments

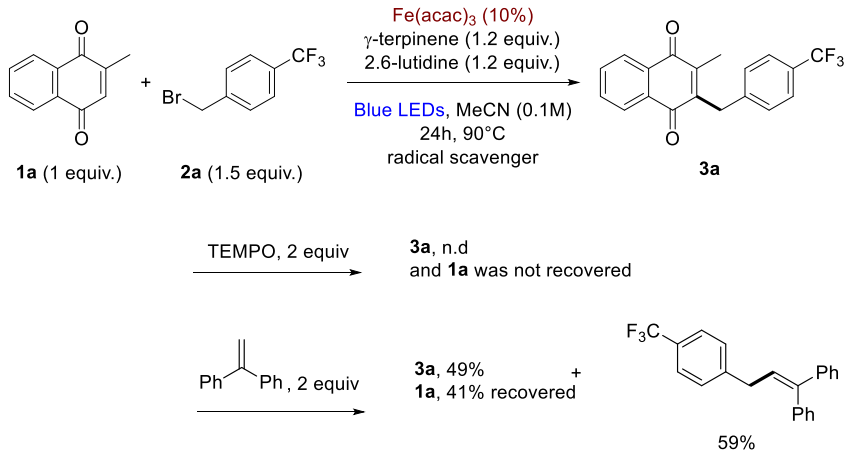

B. $y$-terpinene influence on the reaction yield. ${ }^{a}$

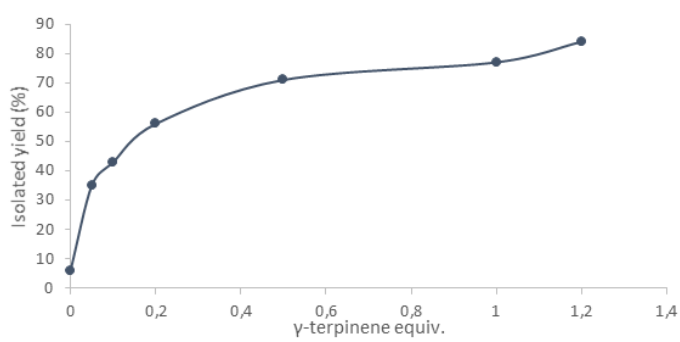

C. Photocatalytic reaction using $\mathrm{PrOH}$ both as solvent and HAT agent.

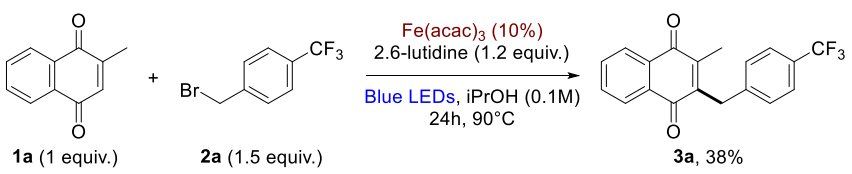

${ }^{a}$ Reaction conditions: 1 (1.0 equiv, $\left.0.5 \mathrm{mmol}\right), \mathbf{2 a}$ (1.5 equiv), 2.6lutidine (1.2 equiv.), $\gamma$-terpinene, $\mathrm{Fe}(\mathrm{acac})_{3}(10 \mathrm{~mol} \%)$, in $\mathrm{CH}_{3} \mathrm{CN}$ at $90^{\circ} \mathrm{C}$ under blue light irradiation during $24 \mathrm{~h}$.

During the optimization (Table S2, entry 2; Table S6, entry 2; Table S7, entry 9 in the Supporting Information), we noticed that both light and iron(III) were essential for the reaction to occur. Alternatively, the absence of a base, HAT agent or oxygen-free atmosphere significantly alters the reaction but quantifiable amounts of the targeted benzylated compounds were still observed. The role of $\gamma$-terpinene as HAT agent was thus investigated. The reaction was thus run in parallel with different concentrations of $\gamma$-terpinene. This demonstrates that the isolated yields do not linearly vary with the $\gamma$-terpinene concentration. A sub-stoichiometric amount of this reactant ( 0.5 equiv) can still efficiently lead to a satisfactory yield of $71 \%$, while its absence only afforded traces of the targeted compound $(\sim 5 \%)$. Even a catalytic amount $(5 \%)$ is quite efficient, affording $35 \%$ of yield. This demonstrates that $\gamma$-terpinene is essential and enhances the reactivity of the reaction (Scheme 4B). Previous reports ${ }^{10}$ have established that quinones, including menadione, can be photo-reduced under light irradiation in the presence of a HAT agent, usually a protic solvent such as isopropanol (iPrOH). Under blue LED irradiation $(420-470 \mathrm{~nm})$, a triplet state species is 
generated and can be quenched by H-donor molecules to afford the semiquinone radical species. Absorption spectrophotometry demonstrated that menadione can be photoactivated by blue LED irradiation thus confirming our hypothesis (See Supporting Information, Figures S1-S6 for details). In contrary, the final 3-benzylmenadione 3a seems not to be involved in such photo-reducing process, as we showed that it did not react under the same conditions (See Supporting Information, Figure S7). As already reported by our group with the NADPH/glutathione reductase/MetHb system, semiquinone radicals can reduce $\mathrm{Fe}$ (III) species into $\mathrm{Fe}$ (II) ones to reinstate the quinone molecule. In aerated media, these semiquinone radicals can alternatively generate superoxide $\mathrm{O}_{2}{ }^{--}$radicals. $^{3 e, 11}$ To support this hypothesis, we set up a model reaction, where $\mathrm{iPrOH}$ plays the role of both the solvent and HAT agent in the absence of $\gamma$-terpinene (Scheme 4C). Very interestingly, in addition to the expected nucleophilic substitution of $\mathrm{iPrOH}$ on benzyl bromide, the benzylated menadione was isolated with a yield of $38 \%$. It is important to mention that the same reaction conducted in the absence of $\gamma$-terpinene in $\mathrm{CH}_{3} \mathrm{CN}$ afforded only traces of $\mathbf{3 a}$ (See Supporting Information, Table S5, entry 2) thus confirming the key role of $\mathrm{iPrOH}$ as a HAT agent. To account for these observations, we propose a putative reaction mechanism based on successive redox cycles (Scheme 5).

Scheme 5. Putative mechanism of the reaction.

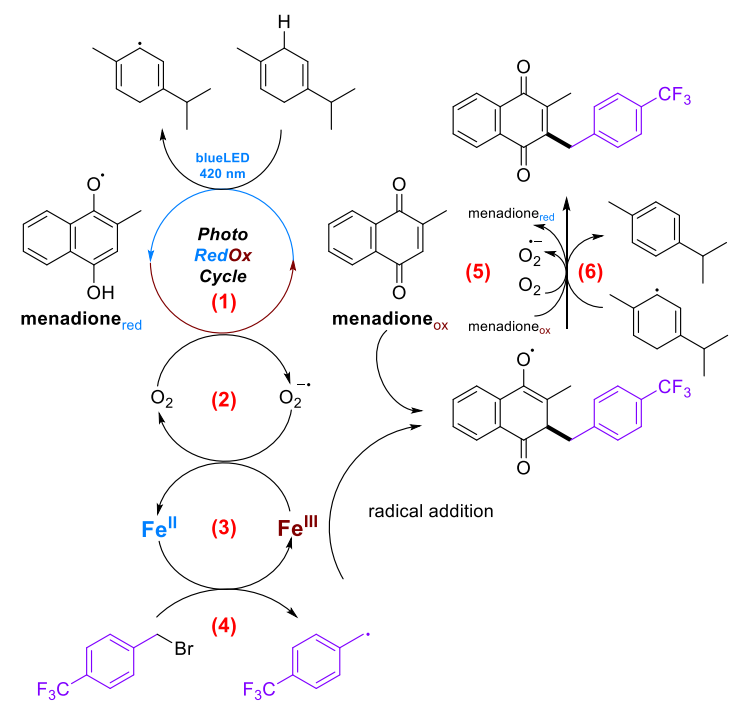

Under blue light irradiation, menadione is excited into its triplet state and quenched by $\gamma$-terpinene (HAT) to afford semiquinone radical in solution (step 1). This radical can thus react in a redox cycle with oxygen (i.e., under anaerobic conditions, the reaction did not take place, Table 1, entry 8) to afford menadione and superoxide radical. $\mathrm{O}_{2}{ }^{--}$is well known to reduce metallic complexes ${ }^{3 e, 12}$, even in natural medium ${ }^{12 d}$ (step 2), which can in turn reduce the C$\mathrm{Br}$ bond of the benzyl bromide to afford the corresponding benzyl radical (step 3). Once this radical generated, addition to menadione takes place to lead to a benzylated semi-quinone radical (step 4). This new radical species can thus react with menadione (or with $\mathrm{O}_{2}$ ) to lead to the targeted benzylated menadione (step 5). The semiquinone or $\mathrm{O}_{2}{ }^{-}$radicals generated during this latter step can be involved in new catalytic cycles (step 2 or 3 ). Semi-quinone intermediate could also be quenched by $\gamma$-terpinene radical in a SET reaction to generate after proton transfer the targeted benzylated quinone (step 6). This was supported by the fact that $p$-cymene was always recovered in the reaction mixtures of benzylated quinones as observed by NMR (Scheme S4 in the Supporting Information). Direct photoreduction of $\mathrm{Fe}(\mathrm{acac})_{3}$ under light irradiation could not be totally excluded (See Supporting Information, Figure S8), and this process has already been reported in the literature. ${ }^{13}$ However, control reactions clearly showed that benzyl bromide was not consumed (e.g., by-products of benzyl radical formation by $\mathrm{Fe}(\mathrm{acac})_{3}$ photoreduction) in the absence of quinone or with a different radical acceptor. Such direct photoreduction process usually occurs via a ligand-to-metal-charge transfer (LMCT) absorption and does not require oxygen contribution. ${ }^{12 d, 13}$ In our system, the absence of oxygen inhibits the menadione benzylation reaction, and different iron sources such as $\mathrm{FeCl}_{3}$, displaying markedly different LMCT absorptions, have been shown to be suitable catalysts for the benzylation reaction.

\section{CONCLUSION}

In conclusion we have developed a convenient, robust and straightforward iron(III) catalyzed photo-redox benzylation of naphthoquinones under blue LED irradiation. A wide variety of polysubstituted benzyl radicals generated from various halides can be efficiently introduced to diversely substituted 1,4-(naphtho)quinones with good yields, thanks to a redox cycle involving the photoreduced (naphtho)quinone, oxygen and ferric complexes. To the best of our knowledge, this is the first time that the photoreactivity of quinone is exploited for its intermolecular functionalization ${ }^{14}$ in a catalytic process.

\section{EXPERIMENTAL SECTION}

\section{General information}

Solvents and reagents: Commercially available starting materials were purchased from Sigma-Aldrich, ABCR GmbH \& Co. KG, Alfa Aesar, Fluorochem and Apollo Scientific and were used without further purification. Solvents were obtained from Sigma-Aldrich and LPCR. All reactions were performed in standard glassware. Thin Layer Chromatography (TLC) were used to monitor reactions (vide infra). Crude mixtures were purified by flash column chromatography. The latter were performed using silica gel 60 (230-400 mesh, 0.040-0.063 mm) purchased from VWR. Monitoring and primary characterization of products were achieved by Thin Layer Chromatography on aluminium sheets coated with silica gel 60 F254 purchased from E. Merck. Eluted TLC's were revealed under UV (325 nm and $254 \mathrm{~nm}$ ) and/or with chemicals.

Nuclear Magnetic Resonance (NMR): The NMR spectra were recorded on a Bruker avance 400 apparatus (1H NMR $400 \mathrm{MHz},{ }^{13} \mathrm{C}$ NMR $101 \mathrm{MHz},{ }^{19} \mathrm{~F}$ NMR $377 \mathrm{MHz}$ ) or Bruker Avance III HD $500 \mathrm{MHz}$ apparatus $\left({ }^{1} \mathrm{H}\right.$ NMR $500 \mathrm{MHz},{ }^{13} \mathrm{C}$ NMR $126 \mathrm{MHz},{ }^{19} \mathrm{~F}$ NMR $471 \mathrm{MHz}$ ) at the ECPM. All chemical shifts $(\delta)$ are quoted in parts per million (ppm). The chemical shifts are referred to the used partial deuterated NMR solvent (for $\mathrm{CDCl}_{3}:{ }^{1} \mathrm{H} \mathrm{NMR}, 7.26 \mathrm{ppm}$ and ${ }^{13} \mathrm{C}$ NMR, $77.16 \mathrm{ppm}$. The coupling constants $(\mathrm{J})$ are given in Hertz $(\mathrm{Hz})$. Resonance patterns are reported with the following notations: br (broad), s (singlet), d (doublet), t (triplet), q (quartet), $\mathrm{m}$ (multiplet), dd (doublet of doublets).

High resolution mass spectrometry (HRMS) analysis were performed with a Bruker MicroTOF mass analyser under ESI in positive ionization mode detection (measurement accuracy $\leq 15 \mathrm{ppm}$ ) at the Service de Spectrométrie de Masse Fédération Chimie Le Bel in Strasbourg.

Photoirradiation reactions were performed using a LED Strip light (Model: 2131, Manufacturer: ADEO SERVICES, 5 meters, 4.8 $\mathrm{W} . \mathrm{m}^{-1}$ ) set to blue irradiation (emission maximum centered on the broadband at $460 \mathrm{~nm}$ ). The light source was placed $10 \mathrm{~cm}$ away from the reaction vessel (borosilicate glass). 


\section{Benzyl halides synthesis}

$\mathbf{2 a}, \mathbf{2 b}, \mathbf{2 i}, \mathbf{2 b}, \mathbf{2 q}$ and $\mathbf{2 a b}$ were obtained from commercial sources.

$\mathbf{2 f}, \mathbf{2} \mathbf{i}$ and $2 \mathbf{a a}$ were obtained from radical bromination of commercial toluenes according to a published procedure. ${ }^{15}$

2c, 2d, 2e, 2g, 2h, 2j, 2k, 2r, 2s, 2u, 2v, 2w, 2x, $2 y$ and $2 \mathrm{z}$ were obtained either from the commercial benzaldehydes, reduced by sodium borohydride, or directly from the commercial benzyl alcohols, and then brominated by triphenylphosphine/dibromide, ${ }^{16}$ triphenylphosphine/tetrabromomethane ${ }^{17}$ or potassium tribromide. ${ }^{18}$

2t was synthetized according to a published procedure starting from the commercially available p-toluidine. ${ }^{19}$

4-trifluoromethylbenzyl iodide $\mathbf{2 a}$ ' was obtained from nucleophilic substitution on $\mathbf{2 a} .^{20}$ 4-trifluoromethylbenzyl chloride $\mathbf{2 a}$ " was obtained from commercial source.

All spectral data were in accordance with published data.

\section{Quinones synthesis}

$4 \mathbf{b}, 4 \mathrm{~h}$ and $4 \mathbf{k}$ were obtained from commercial sources.

$\mathbf{4 a},{ }^{21} \mathbf{4} \mathbf{c},{ }^{22} \mathbf{4 d},{ }^{23} \mathbf{4 e},{ }^{23} \mathbf{4 f},{ }^{24} \mathbf{4} \mathbf{g},{ }^{25} \mathbf{4 i},{ }^{26} \mathbf{4 j}{ }^{2 a}$ were synthetized according to published procedures.

All spectral data were in accordance with published data.

\section{General procedure for the photocatalytic benzylation of quinones}

In a $10 \mathrm{~mL}$ tube, quinone $(0.5 \mathrm{mmol}, 1$ equiv. $)$, benzyl bromide ( $0.75 \mathrm{mmol}, 1.5$ equiv. $)$ and $\mathrm{Fe}(\mathrm{acac})_{3}(0.05 \mathrm{mmol}, 10 \mathrm{~mol} \%)$ were dissolved in Acetonitrile (5 mL). The mixture was stirred and 2,6lutidine ( $0.6 \mathrm{mmol}, 1.2$ equiv.) and $\gamma$-terpinene ( $0.6 \mathrm{mmol}, 1.2$ equiv.) were added successively. The tube was sealed, placed under blue light irradiation and heated up at $90^{\circ} \mathrm{C}$ in an oil bath during 24h. After completion, the mixture was allowed to cool down at room temperature and was partitioned between ethyl acetate (10 $\mathrm{mL})$ and aqueous $1 \mathrm{M} \mathrm{HCl}(10 \mathrm{~mL})$. The aqueous layer was extracted once with $10 \mathrm{~mL}$ ethyl acetate and the reunited organic layers were washed with brine and dried with MgSO4. The solvent was removed and the crude was purified by silica gel chromatography to obtain pure benzylated quinone.

\section{Procedure for the $4 \mathrm{mmol}$ scale photocatalytic benzylation of menadione}

In a $30 \mathrm{~mL}$ tube, quinone (4 mmol, 1 equiv.), 4-trifluoromethylbenzyl bromide $2 \mathrm{a}$ (6 mmol, 1.5 equiv.) and $\mathrm{Fe}(\mathrm{acac})_{3}(0.4 \mathrm{mmol}, 10$ mol\%) were dissolved in Acetonitrile $(20 \mathrm{~mL})$. The mixture was stirred and 2,6-lutidine ( $4.8 \mathrm{mmol}, 1.2$ equiv.) and $\gamma$-terpinene (4.8 mmol, 1.2 equiv.) were added successively. The tube was sealed, placed under blue light irradiation and heated up at $90^{\circ} \mathrm{C}$ in an oil bath during $24 \mathrm{~h}$. After completion, the mixture was allowed to cool down at room temperature and was partitioned between ethyl acetate $(50 \mathrm{~mL})$ and aqueous $1 \mathrm{M} \mathrm{HCl}(50 \mathrm{~mL})$. The aqueous layer was extracted twice with $50 \mathrm{~mL}$ ethyl acetate and the reunited organic layers were washed with brine and dried with $\mathrm{MgSO} 4$. The solvent was removed and the crude was purified by silica gel chromatography $($ Cyclohexane:Toluene $=1: 1, \mathrm{UV})$ to obtain $933 \mathrm{mg}(2.82$ mmol, $71 \%$ yield) of pure benzylated quinone $\mathbf{3 a}$.

\section{Product characterization}

2-methyl-3-(4-(trifluoromethyl)benzoyl)naphthalene-1,4-dione (3a). According to the general procedure, menadione 1a and 4-trifluoromethylbenzyl bromide 2a were used. After chromatography on silica gel (Cyclohexane:Toluene $=1: 1$, UV), $139 \mathrm{mg}(0.42$ mmol, 84\% yield) of 3a were isolated as a yellow solid. m.p: 70$71^{\circ} \mathrm{C} .{ }^{1} \mathbf{H}$ NMR (500 MHz, Chloroform- $\left.\boldsymbol{d}\right) \boldsymbol{\delta} 8.13-8.02(\mathrm{~m}, 2 \mathrm{H})$, $7.74-7.65(\mathrm{~m}, 2 \mathrm{H}), 7.52(\mathrm{~d}, J=8.1 \mathrm{~Hz}, 2 \mathrm{H}), 7.35(\mathrm{~d}, J=8.0 \mathrm{~Hz}$, 2H), 4.08 (s, 2H), 2.25 (s, 3H). ${ }^{19}$ F NMR (471 MHz, Chloroformd) $\boldsymbol{\delta}-62.45 .{ }^{13} \mathbf{C}\left\{{ }^{1} \mathbf{H}\right\}$ NMR (126 MHz, Chloroform-d) $\boldsymbol{\delta} 185.2$, $184.6,145.0,144.5,142.3,133.78,133.76,132.2,132.0,129.0$, $128.9(\mathrm{q}, J=32.3 \mathrm{~Hz}), 126.6,126.5,125.7$ (q, $J=3.8 \mathrm{~Hz}), 124.2$ $(\mathrm{q}, J=271.8 \mathrm{~Hz}), 32.5,13.5$. HRMS (ESI) $\mathbf{m} / \mathbf{z}:[\mathrm{M}+\mathrm{H}]^{+}$Calcd for $\mathrm{C}_{19} \mathrm{H}_{14} \mathrm{~F}_{3} \mathrm{O}_{2}$ 331.0940; Found 331.0949.

2-benzyl-3-methylnaphthalene-1,4-dione (3b). According to the general procedure, menadione 1a and benzyl bromide $\mathbf{2 a}$ were used. After chromatography on silica gel (Cyclohexane:Toluene $=$ 4:6, UV), $90 \mathrm{mg}$ ( $0.34 \mathrm{mmol}, 69 \%$ yield) of $\mathbf{3 b}$ were isolated as a yellow solid. m.p: $105-106^{\circ} \mathrm{C}^{\mathbf{1}}{ }^{\mathbf{H}}$ NMR (400 MHz, Chloroformd) $\delta 8.14-8.03(\mathrm{~m}, 2 \mathrm{H}), 7.70(\mathrm{dd}, J=5.7,3.3 \mathrm{~Hz}, 2 \mathrm{H}), 7.44-6.92$ (m, 5H), $4.04(\mathrm{~s}, 2 \mathrm{H}), 2.25$ (s, 3H). ${ }^{13} \mathbf{C}\left\{{ }^{1} \mathbf{H}\right\}$ NMR (101 MHz, Chloroform- $d$ ) $\delta$ 185.5, 184.8, 145.5, 144.6, 138.2, 133.6, 133.6, $132.3,132.2,128.8,128.7,126.62,126.56,126.4,32.5,13.4$. HRMS (ESI) m/z: $[\mathrm{M}+\mathrm{H}]^{+}$Calcd for $\mathrm{C}_{18} \mathrm{H}_{15} \mathrm{O}_{2}$ 263.1067; Found $263.1072\left(\mathrm{MH}^{+}\right)$.

2-(4-fluorobenzyl)-3-methylnaphthalene-1,4-dione (3c). According to the general procedure, menadione 1a and 4-fluorobenzyl bromide 2c were used. After chromatography on silica gel (Cyclohexane:Toluene $=3: 7, \mathrm{UV}), 93 \mathrm{mg}(0.33 \mathrm{mmol}, 66 \%$ yield $)$ of $\mathbf{3 c}$ were isolated as a yellow solid. m.p: $114-115^{\circ}$ C. ${ }^{1} \mathbf{H}$ NMR (400 MHz, Chloroform- $\boldsymbol{d}) \boldsymbol{\delta} 8.15-8.04(\mathrm{~m}, 2 \mathrm{H}), 7.76-7.66(\mathrm{~m}, 2 \mathrm{H})$, $7.22-7.16(\mathrm{~m}, 2 \mathrm{H}), 7.00-6.90(\mathrm{~m}, 2 \mathrm{H}), 4.00(\mathrm{~s}, 2 \mathrm{H}), 2.25(\mathrm{~s}, 3 \mathrm{H})$. ${ }^{19}$ F NMR (377 MHz, Chloroform- $d$ ) $\delta$-116.52 - -116.64 (m). ${ }^{13} \mathbf{C}\left\{{ }^{1} \mathbf{H}\right\}$ NMR (126 MHz, Chloroform-d) $\boldsymbol{\delta}$ 185.4, 184.7, 161.6 $(\mathrm{d}, J=244.7 \mathrm{~Hz}), 145.2,144.5,133.8(\mathrm{~d}, J=3.3 \mathrm{~Hz}), 133.7,132.2$, $132.1,130.2(\mathrm{~d}, J=7.8 \mathrm{~Hz}), 126.6,126.4,115.6(\mathrm{~d}, J=21.3 \mathrm{~Hz})$, 31.8, 13.4. HRMS (ESI) $\mathbf{m} / \mathbf{z}:[\mathrm{M}+\mathrm{H}]^{+}$Calcd for $\mathrm{C}_{18} \mathrm{H}_{14} \mathrm{FO}_{2}$ 281.0972; Found 281.0987.

2-(4-chlorobenzyl)-3-methylnaphthalene-1,4-dione (3d). According to the general procedure, menadione $\mathbf{1 a}$ and 4-chlorobenzyl bromide 2d were used. After chromatography on silica gel (Cyclohexane:Toluene $=4: 6$, UV), $109 \mathrm{mg}(0.37 \mathrm{mmol}, 73 \%$ yield $)$ of 3d were isolated as a yellow solid. m.p: $132-133^{\circ} \mathrm{C}$. ${ }^{\mathbf{1}} \mathbf{H}$ NMR (400 MHz, Chloroform- $\boldsymbol{d}$ ) $\boldsymbol{\delta} 8.28-7.95(\mathrm{~m}, 2 \mathrm{H}), 7.70(\mathrm{~m}, 2 \mathrm{H}), 7.25-$ $7.20(\mathrm{~m}, 2 \mathrm{H}), 7.19-7.13(\mathrm{~m}, 2 \mathrm{H}), 3.99(\mathrm{~s}, 1 \mathrm{H}), 2.24(\mathrm{~s}, 1 \mathrm{H})$. ${ }^{13} \mathbf{C}\left\{{ }^{1} \mathbf{H}\right\}$ NMR (101 MHz, Chloroform- $\left.d\right) \delta$ 185.4, 184.7, 145.0, $144.7,136.7,133.7,132.4,132.2,132.1,130.1,128.9,126.6$, 126.5, 32.0, 13.4. HRMS (ESI) m/z: $[\mathrm{M}+\mathrm{H}]^{+}$Calcd for $\mathrm{C}_{18} \mathrm{H}_{14} \mathrm{ClO}_{2}$ 297.0677; Found 297.0684.

2-(4-bromobenzyl)-3-methylnaphthalene-1,4-dione (3e). According to the general procedure, menadione 1a and 4-bromobenzyl bromide 2e were used. After chromatography on silica gel (Cyclohexane:Toluene $=4: 6, \mathrm{UV}), 137 \mathrm{mg}(0.402 \mathrm{mmol}, 80 \%$ yield $)$ of $\mathbf{3 e}$ were isolated as a yellow solid. m.p: $123-124^{\circ} \mathrm{C} .{ }^{\mathbf{1}} \mathbf{H}$ NMR (400 MHz, Chloroform- $\boldsymbol{d}$ ) $\boldsymbol{\delta} 8.19-8.01(\mathrm{~m}, 2 \mathrm{H}), 7.70(\mathrm{~m}, 2 \mathrm{H})$, $7.44-7.35(\mathrm{~m}, 2 \mathrm{H}), 7.16-7.04(\mathrm{~m}, 2 \mathrm{H}), 3.97(\mathrm{~s}, 2 \mathrm{H}), 2.24(\mathrm{~s}, 3 \mathrm{H})$. ${ }^{13} \mathbf{C}\left\{{ }^{1} \mathbf{H}\right\}$ NMR (101 MHz, Chloroform-d) $\delta$ 185.3, 184.7, 144.9, $144.7,137.2,133.7,132.2,132.1,131.9,130.5,126.6,126.5$, 120.4, 32.0, 13.4. HRMS (ESI) m/z: $[\mathrm{M}+\mathrm{H}]^{+}$Calcd for $\mathrm{C}_{18} \mathrm{H}_{14} \mathrm{BrO}_{2}$ 341.0172; Found $341.0180\left(\mathrm{MH}^{+}\right)$.

2-(4-iodobenzyl)-3-methylnaphthalene-1,4-dione (3f). According to the general procedure, menadione 1a and 4-iodobenzyl bromide $2 \mathrm{e}$ were used. After chromatography on silica gel (Cyclohexane:Toluene $=4: 6, \mathrm{UV}), 158 \mathrm{mg}(0.407 \mathrm{mmol}, 81 \%$ yield $)$ of $\mathbf{3 f}$ were isolated as a yellow solid. m.p: $121-122^{\circ} \mathrm{C} .{ }^{1} \mathbf{H}$ NMR (400 MHz, Chloroform- $\boldsymbol{d}$ ) $\boldsymbol{\delta} 8.18-7.99(\mathrm{~m}, 2 \mathrm{H}), 7.79-7.64(\mathrm{~m}, 2 \mathrm{H})$, $7.60-7.47$ (m, 2H), $7.03-6.86(\mathrm{~m}, 2 \mathrm{H}), 3.97$ (s, 2H), 2.24 (s, 3H). ${ }^{13} \mathbf{C}\left\{{ }^{1} \mathbf{H}\right\}$ NMR (101 MHz, Chloroform-d) $\delta$ 185.4, 184.7, 144.9, $144.7,137.9,137.8,133.7,132.2,132.1,130.8,126.7,126.5,91.8$, 
32.2, 13.5. HRMS (ESI) $\mathbf{m} / \mathbf{z}:[\mathrm{M}+\mathrm{H}]^{+}$Calcd for $\mathrm{C}_{18} \mathrm{H}_{14} \mathrm{IO}_{2}$ : 389.0033; Found 389.0043 .

2-methyl-3-(4-nitrobenzyl)naphthalene-1,4-dione (3g). According to the general procedure, menadione 1a and 4-nitrobenzyl bromide $2 \mathrm{~g}$ were used. After chromatography on silica gel (Cyclohexane:Ethyl acetate $=9: 1, \mathrm{UV}), 118 \mathrm{mg}(0.38 \mathrm{mmol}, 77 \%$ yield $)$ of $3 \mathrm{~g}$ were isolated as a yellow solid. m.p: $153-154^{\circ} \mathrm{C} .{ }^{1} \mathbf{H}$ NMR (400 MHz, Chloroform- $\boldsymbol{d}) \boldsymbol{\delta} 8.09-7.96(\mathrm{~m}, 4 \mathrm{H}), 7.70-7.60(\mathrm{~m}, 2 \mathrm{H})$, $7.38-7.28(\mathrm{~m}, 2 \mathrm{H}), 4.06$ (s, 2H), 2.19 (s, 3H). ${ }^{13} \mathbf{C}\left\{{ }^{1} \mathbf{H}\right\}$ NMR $(101$ MHz, Chloroform- $d$ ) $\delta$ 185.1, 184.5, 146.8, 146.0, 145.3, 144.0, 134.0, 133.9, 132.2, 131.9, 129.5, 126.7, 126.6, 124.0, 32.6, 13.6. HRMS (ESI) m/z: $[\mathrm{M}+\mathrm{H}]^{+}$Calcd for $\mathrm{C}_{18} \mathrm{H}_{14} \mathrm{NO}_{4} 308.0917$; Found 308.0923 .

\section{4-(3-methyl-1,4-dioxo-1,4-dihydronaphthalene-2-car-}

bonyl)benzonitrile (3h). According to the general procedure, menadione 1a and 4-cyannobenzyl bromide $\mathbf{2 h}$ were used. After chromatography on silica gel (Toluene, UV), $108 \mathrm{mg}(0.38 \mathrm{mmol}, 75 \%$ yield) of $\mathbf{3 h}$ were isolated as a yellow solid. m.p: $161-162{ }^{\circ} \mathrm{C} .{ }^{1} \mathbf{H}$ NMR (400 MHz, Chloroform-d) $\delta 8.15-8.01$ (m, 2H), $7.82-$ $7.66(\mathrm{~m}, 2 \mathrm{H}), 7.63-7.50(\mathrm{~m}, 2 \mathrm{H}), 7.41-7.30(\mathrm{~m}, 2 \mathrm{H}), 4.08(\mathrm{~s}$, 2H), 2.24 (s, 3H). ${ }^{13} \mathbf{C}\left\{{ }^{1} \mathbf{H}\right\}$ NMR (101 MHz, Chloroform-d) $\delta$ $185.1,184.6,145.2$, 144.0, 143.6, 133.9, 133.9, 132.6, 132.2, 132.0, 129.5, 126.7, 126.6, 118.9, 110.6, 32.8, 13.6. HRMS (ESI) $\mathbf{m} / \mathbf{z}:[\mathrm{M}+\mathrm{H}]^{+}$Calcd for $\mathrm{C}_{19} \mathrm{H}_{14} \mathrm{NO}_{2} 288.1019$; Found 288.1033.

methyl 4-((3-methyl-1,4-dioxo-1,4-dihydronaphthalen-2yl)methyl)benzoate (3i). According to the general procedure, menadione $1 \mathbf{a}$ and methyl 4-(bromomethyl)benzoate $\mathbf{2 i}$ were used. After chromatography on silica gel (Toluene, UV), $80 \mathrm{mg}(0.25$ mmol, $50 \%$ yield) of $\mathbf{3 i}$ were isolated as a yellow solid. m.p: 111$112^{\circ} \mathrm{C}$. ${ }^{1} \mathbf{H}$ NMR (400 MHz, Chloroform-d) $\delta 8.12-8.06(\mathrm{~m}$, $2 \mathrm{H}), 7.95-7.91(\mathrm{~m}, 2 \mathrm{H}), 7.74-7.68(\mathrm{~m}, 2 \mathrm{H}), 7.29(\mathrm{~d}, \mathrm{~J}=8.5 \mathrm{~Hz}$, 2H), 4.08 (s, 2H), 3.88 (s, 3H), 2.23 (s, 3H). ${ }^{13} \mathbf{C}\left\{{ }^{1} \mathbf{H}\right\}$ NMR (101 MHz, Chloroform-d) $\delta$ 185.3, 184.6, 167.0, 145.0, 144.7, 143.6, 133.8, 132.2, 132.1, 130.1, 128.7, 128.6, 126.7, 126.5, 52.2, 32.7, 13.5. HRMS (ESI) $\mathbf{m} / \mathbf{z}:[\mathrm{M}+\mathrm{H}]^{+}$Calcd for $\mathrm{C}_{20} \mathrm{H}_{17} \mathrm{O}_{4} 321.1121$; Found 321.1108 .

2-methyl-3-(4-(trifluoromethoxy)benzyl)naphthalene-1,4-dione $(3 \mathbf{j})$. According to the general procedure, menadione 1a and 4trifluoromethoxybenzyl bromide $\mathbf{3 j}$ were used. After chromatography on silica gel (Cyclohexane:Toluene $=4: 6, \mathrm{UV}), 138 \mathrm{mg}(0.4$ mmol, $80 \%$ yield) of $\mathbf{3 j}$ were isolated as a yellow solid. m.p: $65-$ $66^{\circ} \mathrm{C} .{ }^{1} \mathrm{H}$ NMR (400 MHz, Chloroform- $\left.\boldsymbol{d}\right) \boldsymbol{\delta} 8.12-8.05(\mathrm{~m}, 2 \mathrm{H})$, $7.71(\mathrm{dd}, J=5.8,3.3 \mathrm{~Hz}, 2 \mathrm{H}), 7.30-7.22(\mathrm{~m}, 2 \mathrm{H}), 7.11(\mathrm{dt}, J=$ 7.7, $1.0 \mathrm{~Hz}, 2 \mathrm{H}), 4.03$ (s, 2H), 2.26 (s, 3H). ${ }^{19}$ F NMR (377 MHz, Chloroform-d) $\delta-57.93 .{ }^{13} \mathbf{C}\left\{{ }^{1} \mathbf{H}\right\}$ NMR (101 MHz, Chloroformd) $\delta 185.4,184.7,147.9(\mathrm{q}, J=2.0 \mathrm{~Hz}), 144.9,144.7,136.9,133.8$, $132.2,132.1,130.0,129.2,128.4,126.7,126.5,120.6(\mathrm{~d}, J=256.9$ $\mathrm{Hz}$ ), 32.0, 13.5. HRMS (ESI) $\mathbf{m} / \mathbf{z}:[\mathrm{M}+\mathrm{H}]^{+}$Calcd for $\mathrm{C}_{19} \mathrm{H}_{14} \mathrm{~F}_{3} \mathrm{O}_{3}$ 347.0890; Found 347.0870.

2-methyl-3-(4-methylbenzyl)naphthalene-1,4-dione (3k). According to the general procedure, menadione 1a and 4-methylbenzyl bromide 2k were used. After chromatography on silica gel (Cyclohexane:Toluene $=4: 6, \mathrm{UV}), 71 \mathrm{mg}(0.26 \mathrm{mmol}, 51 \%$ yield $)$ of 3k were isolated as a yellow solid. m.p: $135-136{ }^{\circ} \mathbf{C} .{ }^{\mathbf{1}} \mathbf{H}$ NMR (500 MHz, Chloroform- $\boldsymbol{d}) \boldsymbol{\delta} 8.08(\mathrm{~m}, 2 \mathrm{H}), 7.69(\mathrm{~m}, 2 \mathrm{H}), 7.13(\mathrm{~d}, J=$ $8.2 \mathrm{~Hz}, 2 \mathrm{H}), 7.07(\mathrm{~d}, J=8.1 \mathrm{~Hz}, 2 \mathrm{H}), 4.00(\mathrm{~s}, 2 \mathrm{H}), 2.29(\mathrm{~s}, 3 \mathrm{H})$, 2.25 (s, 3H). ${ }^{13} \mathbf{C}\left\{{ }^{1} \mathbf{H}\right\}$ NMR (126 MHz, Chloroform-d) $\delta$ 185.6, $184.8,145.7,144.4,136.1,135.1,133.59,133.55,132.3,132.2$, 129.5, 128.6, 126.6, 126.4, 32.1, 21.1, 13.4. HRMS (ESI) m/z: $[\mathrm{M}+\mathrm{H}]^{+}$Calcd for $\mathrm{C}_{19} \mathrm{H}_{17} \mathrm{O}_{2} 277.1223$; Found 277.1227.

2-methyl-3-(3-(trifluoromethyl)benzyl)naphthalene-1,4-dione (31). According to the general procedure, menadione 1a and 3-trifluoromethylbenzyl bromide $\mathbf{2 l}$ were used. After chromatography on silica gel (Cyclohexane:Toluene $=4: 6, \mathrm{UV}), 126 \mathrm{mg}(0.38$ mmol, $76 \%$ yield) of $\mathbf{3 l}$ were isolated as a yellow solid. m.p: $92-93$ ${ }^{\circ} \mathrm{C}^{1}{ }^{1} \mathrm{H}$ NMR (400 MHz, Chloroform-d) $\boldsymbol{\delta} 8.09$ (m, 2H), 7.71 (m, 2H), $7.53-7.34(\mathrm{~m}, 4 \mathrm{H}), 4.08(\mathrm{~s}, 2 \mathrm{H}), 2.25$ (s, 3H). ${ }^{19}$ F NMR (377 MHz, Chloroform- $d$ ) $\delta-62.58 .{ }^{13} \mathbf{C}\left\{{ }^{1} \mathbf{H}\right\}$ NMR (101 MHz, Chloroform- $d) \delta 185.1,184.5,144.9,144.4,139.1,133.6,132.1$, 131.94, 131.92, 131.91, $131.0(\mathrm{q}, J=32.2 \mathrm{~Hz}), 129.1,126.6,126.4$, $125.3(\mathrm{q}, J=3.9 \mathrm{~Hz}), 124.0(\mathrm{q}, J=272.4 \mathrm{~Hz}), 123.4(\mathrm{q}, J=3.8 \mathrm{~Hz})$, 32.3, 13.4. HRMS (ESI) $\mathbf{m} / \mathbf{z}:[\mathrm{M}+\mathrm{H}]^{+}$Calcd for $\mathrm{C}_{19} \mathrm{H}_{14} \mathrm{~F}_{3} \mathrm{O}_{2}$ 331.0940; Found 331.0936.

2-methyl-3-(2-(trifluoromethyl)benzyl)naphthalene-1,4-dione $(\mathbf{3 m})$. According to the general procedure, menadione 1a and 2trifluoromethylbenzyl bromide $\mathbf{2 m}$ were used. After chromatography on silica gel (Cyclohexane:Toluene $=4: 6, \mathrm{UV}), 127 \mathrm{mg}(0.38$ mmol, 77\% yield) of $\mathbf{3 m}$ were isolated as a yellow solid. m.p: 106$107^{\circ} \mathrm{C}^{1}{ }^{1} \mathrm{H}$ NMR (400 MHz, Chloroform- $\left.\boldsymbol{d}\right) \boldsymbol{\delta} 8.28-8.01$ (m, 2H), $7.78-7.72(\mathrm{~m}, 2 \mathrm{H}), 7.70(\mathrm{~d}, J=7.3 \mathrm{~Hz}, 1 \mathrm{H}), 7.36(\mathrm{t}, J=7.1 \mathrm{~Hz}$, $1 \mathrm{H}), 7.30(\mathrm{t}, J=7.3 \mathrm{~Hz}, 1 \mathrm{H}), 6.96(\mathrm{~d}, J=7.6 \mathrm{~Hz}, 1 \mathrm{H}), 4.25(\mathrm{~s}, 2 \mathrm{H})$, 2.11 (s, 3H). ${ }^{19} \mathbf{F}$ NMR (377 MHz, Chloroform-d) $\delta-60.90$. ${ }^{13} \mathbf{C}\left\{{ }^{1} \mathbf{H}\right\}$ NMR (101 MHz, Chloroform-d) $\delta$ 185.1, 184.5, 146.4, $144.4,136.8,133.83,133.81,132.4,132.2,132.1,128.7$ (q, $J=$ $29.9 \mathrm{~Hz}), 128.3,126.8,126.60,126.56,126.4(\mathrm{q}, J=5.8 \mathrm{~Hz}), 124.7$ (q, $J=273.8 \mathrm{~Hz}), 28.8(\mathrm{q}, J=2.6 \mathrm{~Hz}), 13.2$. HRMS (ESI) m/z: $[\mathrm{M}+\mathrm{H}]^{+}$Calcd for $\mathrm{C}_{19} \mathrm{H}_{14} \mathrm{~F}_{3} \mathrm{O}_{2}$ 331.0940; Found 331.0930.

2-methyl-3-(2-methylbenzyl)naphthalene-1,4-dione (3n). According to the general procedure, menadione 1a and 2-methylbenzyl bromide 2n were used. After chromatography on silica gel (Cyclohexane:Toluene $=4: 6, \mathrm{UV}), 92 \mathrm{mg}(0.33 \mathrm{mmol}, 67 \%$ yield $)$ of 3n were isolated as a yellow solid. m.p: $61-62{ }^{\circ} \mathrm{C} .{ }^{\mathbf{1}} \mathbf{H}$ NMR (400 MHz, Chloroform- $\boldsymbol{d}) \boldsymbol{\delta} 8.15-8.11(\mathrm{~m}, 1 \mathrm{H}), 8.11-8.06(\mathrm{~m}, 1 \mathrm{H})$, $7.77-7.67(\mathrm{~m}, 2 \mathrm{H}), 7.20(\mathrm{dd}, J=7.3,1.6 \mathrm{~Hz}, 1 \mathrm{H}), 7.11(\mathrm{td}, J=$ 7.4, $1.5 \mathrm{~Hz}, 1 \mathrm{H}), 7.04(\mathrm{td}, J=7.6,1.6 \mathrm{~Hz}, 1 \mathrm{H}), 6.84(\mathrm{dd}, J=7.6$, $1.3 \mathrm{~Hz}, 1 \mathrm{H}), 3.97(\mathrm{~s}, 2 \mathrm{H}), 2.45$ (s, 3H), $2.16(\mathrm{~s}, 3 \mathrm{H}) .{ }^{13} \mathbf{C}\left\{{ }^{\mathbf{1}} \mathbf{H}\right\} \mathbf{N M R}$ (101 MHz, Chloroform-d) $\delta$ 185.3, 184.6, 145.7, 145.5, 136.4, $136.1,133.7,133.6,132.4,132.3,130.5,126.73,126.68,126.5$, 126.5, 126.3, 29.7, 20.1, 13.4. HRMS (ESI) m/z: $[\mathrm{M}+\mathrm{H}]^{+}$Calcd for $\mathrm{C}_{19} \mathrm{H}_{17} \mathrm{O}_{2} 277.1223$; Found 277.1224.

2-(2-bromobenzyl)-3-methylnaphthalene-1,4-dione (3o). According to the general procedure, menadione 1a and 2-bromobenzyl bromide 20 were used. After chromatography on silica gel (Cyclohexane:Toluene $=4: 6, \mathrm{UV}), 134 \mathrm{mg}(0.39 \mathrm{mmol}, 79 \%$ yield $)$ of 3o were isolated as a yellow solid. m.p: $102-103^{\circ} \mathrm{C}$. ${ }^{\mathbf{1}} \mathbf{H}$ NMR (400 MHz, Chloroform- $\boldsymbol{d}) \delta 8.19-7.99(\mathrm{~m}, 2 \mathrm{H}), 7.79-7.64(\mathrm{~m}, 2 \mathrm{H})$, $7.59(\mathrm{dd}, J=8.0,1.4 \mathrm{~Hz}, 1 \mathrm{H}), 7.15(\mathrm{td}, J=7.5,1.4 \mathrm{~Hz}, 1 \mathrm{H}), 7.06$ $(\mathrm{td}, J=7.6,1.8 \mathrm{~Hz}, 1 \mathrm{H}), 6.92(\mathrm{dd}, J=7.6,1.8 \mathrm{~Hz}, 1 \mathrm{H}), 4.13(\mathrm{~s}$, 2H), 2.13 (s, 3H). ${ }^{13} \mathbf{C}\left\{{ }^{1} \mathbf{H}\right\}$ NMR (101 MHz, Chloroform-d) $\boldsymbol{\delta}$ 185.2 , 184.5, 146.0, 144.7, 137.5, 133.8, 133.7, 133.1, 132.3, 132.2, 128.8, 128.1, 127.7, 126.7, 126.5, 124.8, 32.8, 13.5. HRMS (ESI) $\mathbf{m} / \mathbf{z}:[\mathrm{M}+\mathrm{H}]^{+}$Calcd for $\mathrm{C}_{18} \mathrm{H}_{14} \mathrm{BrO}_{2}$ 341.0172; Found 341.0181 .

2-(3,5-dimethylbenzyl)-3-methylnaphthalene-1,4-dione (3p). According to the general procedure, menadione 1a and 3,5-dimethylbenzyl bromide $\mathbf{2 p}$ were used. After chromatography on silica gel (Cyclohexane:Toluene = 4:6, UV), $101 \mathrm{mg}(0.35 \mathrm{mmol}, 70 \%$ yield) of $\mathbf{3 p}$ were isolated as a yellow solid. m.p: $108-109^{\circ} \mathbf{C} .{ }^{\mathbf{1}} \mathbf{H}$ NMR (400 MHz, Chloroform- $\boldsymbol{d}$ ) $\boldsymbol{\delta} 8.15$ - 8.00 (m, 2H), 7.70 (dd, $J=5.8,3.3 \mathrm{~Hz}, 2 \mathrm{H}), 7.02-6.63(\mathrm{~m}, 3 \mathrm{H}), 3.96(\mathrm{~s}, 2 \mathrm{H}), 2.26(\mathrm{~s}, 6 \mathrm{H})$, 2.25 (s, 3H). ${ }^{13} \mathbf{C}\left\{{ }^{1} \mathbf{H}\right\}$ NMR (101 MHz, Chloroform-d) $\delta$ 185.6, $184.9,145.7,144.5,138.3,138.0,133.6,133.6,132.32$, 132.25, 128.3, 126.7, 126.5, 126.4, 32.4, 21.4, 13.5. HRMS (ESI) $\mathbf{m} / \mathbf{z}$ : $[\mathrm{M}+\mathrm{H}]^{+}$Calcd for $\mathrm{C}_{20} \mathrm{H}_{19} \mathrm{O}_{2} 291.1380$; Found 291.1377. 
2-(3,5-dimethoxybenzyl)-3-methylnaphthalene-1,4-dione (3q). According to the general procedure, menadione 1a and 3,5-dimethoxybenzyl bromide $\mathbf{2 q}$ were used. After chromatography on silica gel (Cyclohexane:Ethyl Acetate $=95: 5, \mathrm{UV}), 110 \mathrm{mg}(0.34$ mmol, $68 \%$ yield) of $\mathbf{3 q}$ were isolated as a yellow solid. m.p: 133$134{ }^{\circ} \mathrm{C} .{ }^{1} \mathbf{H}$ NMR (400 MHz, Chloroform-d) $\boldsymbol{\delta} 8.17$ - 7.99 (m, $2 \mathrm{H}), 7.74-7.65(\mathrm{~m}, 2 \mathrm{H}), 6.37(\mathrm{~d}, J=2.2 \mathrm{~Hz}, 2 \mathrm{H}), 6.29$ (t, $J=2.2$ $\mathrm{Hz}, 1 \mathrm{H}), 3.97$ (s, 2H), 3.75 (s, 6H), 2.24 (s, 3H). ${ }^{13} \mathbf{C}\left\{{ }^{\mathbf{1}} \mathbf{H}\right\}$ NMR (101 MHz, Chloroform-d) $\delta$ 185.5, 184.7, 161.0, 145.1, 144.8, 140.4, 133.61, 133.59, 132.3, 132.2, 126.6, 126.4, 107.0, 98.1, 55.4, 32.6, 13.4. HRMS (ESI) $\mathbf{m} / \mathbf{z}:[\mathrm{M}+\mathrm{H}]^{+}$Calcd for $\mathrm{C}_{20} \mathrm{H}_{19} \mathrm{O}_{4}$ 323.1278; Found 323.1287.

2-(3,5-dibromobenzyl)-3-methylnaphthalène-1,4-dione (3r). According to the general procedure, menadione 1a and 3,5-dibromobenzyl bromide $\mathbf{2 r}$ were used. After chromatography on silica gel $($ Cyclohexane:Toluene = 1:1, UV), $135 \mathrm{mg}(0.32 \mathrm{mmol}, 64 \%$ yield) of $3 \mathbf{r}$ were isolated as a yellow solid. m.p: $151-152^{\circ} \mathrm{C} .{ }^{1} \mathbf{H}$ (400MHz, Chloroform-d) $\delta$ 8.11 (m, 2H), 7.75-7.71 (m, 2H), 7.51 (t, J = 1.8 Hz, 1H), $7.30(\mathrm{~m}, 2 \mathrm{H}), 3.91(\mathrm{~s}, 2 \mathrm{H}), 2.23(\mathrm{~s}, 3 \mathrm{H}) .{ }^{13} \mathbf{C}\left\{{ }^{1} \mathbf{H}\right\}$ NMR (126 MHz, Chloroform-d) $\delta$ 185.2, 184.5, 145.3, 143.9, $142.1,133.88,133.86,132.5,132.2,132.0,130.5,126.8,126.6$, 123.3, 32.0, 13.6. HRMS (ESI) $\mathbf{m} / \mathbf{z}:[\mathrm{M}+\mathrm{H}]^{+}$Calcd for $\mathrm{C}_{18} \mathrm{H}_{13} \mathrm{Br}_{2} \mathrm{O}_{2}$ 418.9277; Found 418.9290.

2-(3,5-diiodobenzyl)-3-methylnaphthalène-1,4-dione (3s). According to the general procedure, menadione 1a and 3,5-diiodobenzyl bromide $2 \mathrm{~s}$ were used. After chromatography on silica gel (Cyclohexane:Toluene $=1: 1, \mathrm{UV}), 191 \mathrm{mg}(0.37 \mathrm{mmol}, 74 \%$ yield $)$ of $3 \mathrm{~s}$ were isolated as a yellow solid, along with $5 \%$ non separable contaminating material. m.p: $176-177^{\circ} \mathrm{C} .{ }^{\mathbf{1}} \mathbf{H}(\mathbf{4 0 0 M H z}$, Chloroform- $\boldsymbol{d}) \boldsymbol{\delta} 8.12(\mathrm{~m}, 2 \mathrm{H}), 7.88(\mathrm{t}, \mathrm{J}=1.8 \mathrm{~Hz}, 1 \mathrm{H}), 7.72(\mathrm{~m}, 2 \mathrm{H})$, 7.53-7.50 (m, 2H), 3.91 (s, 2H), 2.23 (s, 3H). ${ }^{13} \mathbf{C}\left\{{ }^{1} \mathbf{H}\right\}$ NMR (126 MHz, Chloroform- $d$ ) $\delta$ 185.2, 184.5, 145.2, 143.9, 143.5, 142.3, 136.9, 133.9, 133.8, 132.2, 132.0, 126.8, 126.6, 95.2, 31.7, 13.6. HRMS (ESI) m/z: $[\mathrm{M}+\mathrm{H}]^{+}$Calcd for $\mathrm{C}_{18} \mathrm{H}_{13} \mathrm{I}_{2} \mathrm{O}_{2}$ 514.9000; Found 514.8989 .

2-(2,6-dichlorobenzyl)-3-methylnaphthalene-1,4-dione (3t). According to the general procedure, menadione 1a and 2,6-dichlorobenzyl bromide $\mathbf{2 t}$ were used. After chromatography on silica gel $($ Cyclohexane:Toluene $=4: 6, \mathrm{UV}), 126 \mathrm{mg}(0.38 \mathrm{mmol}, 76 \%$ yield $)$ of $3 \mathbf{t}$ were isolated as a yellow solid. m.p: $203-204{ }^{\circ} \mathrm{C} .{ }^{1} \mathbf{H}$ NMR (400 MHz, Chloroform- $d$ ) $\delta 8.21-7.99$ (m, 2H), $7.75-7.63$ (m, $2 \mathrm{H}), 7.30(\mathrm{~d}, J=8.0 \mathrm{~Hz}, 2 \mathrm{H}), 7.15-7.08(\mathrm{~m}, 1 \mathrm{H}), 4.37(\mathrm{~s}, 2 \mathrm{H})$, 2.04 (s, 3H). ${ }^{13} \mathbf{C}\left\{{ }^{1} \mathbf{H}\right\}$ NMR (101 MHz, Chloroform-d) $\boldsymbol{\delta} 185.1$, $184.1,144.6,144.5,135.9,135.5,133.6,133.5,132.24,132.20$, 128.6, 128.3, 126.8, 126.4, 29.5, 12.9. HRMS (ESI) m/z: $[\mathrm{M}+\mathrm{H}]^{+}$ Calcd for $\mathrm{C}_{18} \mathrm{H}_{13} \mathrm{Cl}_{2} \mathrm{O}_{2} 331.0287$; Found 331.0280.

2-(2,5-dimethoxybenzyl)-3-methylnaphthalene-1,4-dione (3u). According to the general procedure, menadione 1a and 2,5-dimethoxybenzyl bromide $\mathbf{2 u}$ were used. After chromatography on silica gel (Toluene, UV), $128 \mathrm{mg}(0.39 \mathrm{mmol}, 78 \%$ yield) of $\mathbf{3 u}$ were isolated as a yellow solid. m.p: $148-149{ }^{\circ} \mathrm{C} .{ }^{\mathbf{1}} \mathbf{H}$ NMR (400 MHz, Chloroform- $\boldsymbol{d}) \boldsymbol{\delta} 8.14-8.01(\mathrm{~m}, 2 \mathrm{H}), 7.80-7.61(\mathrm{~m}, 2 \mathrm{H})$, $6.78(\mathrm{~d}, J=8.8 \mathrm{~Hz}, 1 \mathrm{H}), 6.69(\mathrm{dd}, J=8.8,3.1 \mathrm{~Hz}, 1 \mathrm{H}), 6.62(\mathrm{~d}, J$ $=3.0 \mathrm{~Hz}, 1 \mathrm{H}), 4.00(\mathrm{~s}, 2 \mathrm{H}), 3.80(\mathrm{~s}, 3 \mathrm{H}), 3.69(\mathrm{~s}, 3 \mathrm{H}), 2.17(\mathrm{~s}, 3 \mathrm{H})$. ${ }^{13} \mathbf{C}\left\{{ }^{1} \mathbf{H}\right\}$ NMR (101 MHz, Chloroform-d) $\delta$ 185.5, 184.7, 153.7, 151.7, 145.6, 145.1, 133.54, 133.47, 132.38, 132.37, 127.8, 126.6, 126.4, 116.4, 111.3, 111.1, 56.1, 55.8, 26.9, 13.2. HRMS (ESI) $\mathbf{m} / \mathbf{z}:[\mathrm{M}+\mathrm{H}]^{+}$Calcd for $\mathrm{C}_{20} \mathrm{H}_{19} \mathrm{O}_{4}: 323.1278$; Found 323.1286.

2-(3-fluoro-4-nitrobenzyl)-3-methylnaphthalene-1,4-dione (3v). According to the general procedure, menadione 1a and 4-nitro-3-fluorobenzyl bromide $\mathbf{2 v}$ were used. After chromatography on silica gel (Cyclohexane:Toluene $=4: 6, \mathrm{UV}), 91 \mathrm{mg}(0.28 \mathrm{mmol}$, $56 \%$ yield) of $\mathbf{3 v}$ were isolated as a yellow solid. m.p: $140-141^{\circ} \mathrm{C}$.
${ }^{1}$ H NMR (400 MHz, Chloroform-d) $\delta 8.16$ - 8.05 (m, 2H), 7.99 $(\mathrm{dd}, J=8.7,7.7 \mathrm{~Hz}, 1 \mathrm{H}), 7.82-7.65(\mathrm{~m}, 2 \mathrm{H}), 7.22-7.11(\mathrm{~m}, 2 \mathrm{H})$, 4.10 (s, 2H), 2.26 (s, 3H). ${ }^{19}$ F NMR (377 MHz, Chloroform-d) $\delta$ -116.59 (m). ${ }^{13} \mathbf{C}\left\{{ }^{1} \mathbf{H}\right\}$ NMR (101 MHz, Chloroform-d) $\delta$ 184.9, $184.4,155.8(\mathrm{~d}, J=265.6 \mathrm{~Hz}), 147.7(\mathrm{~d}, J=8.0 \mathrm{~Hz}), 145.6,143.2$, $135.9,134.1,134.0,132.2,131.9,126.7(\mathrm{~d}, J=4.0 \mathrm{~Hz}), 126.6(\mathrm{~d}, J$ $=2.5 \mathrm{~Hz}), 124.80,124.76,118.5(\mathrm{~d}, J=21.3 \mathrm{~Hz}), 32.6(\mathrm{~d}, J=1.4$ $\mathrm{Hz}$ ), 13.6. HRMS (ESI) $\mathbf{m} / \mathbf{z}:[\mathrm{M}+\mathrm{H}]^{+}$Calcd for $\mathrm{C}_{18} \mathrm{H}_{13} \mathrm{FNO}_{4}$ 326.0823; Found 326.0802 .

2-(2-chloro-5-nitrobenzyl)-3-methylnaphthalene-1,4-dione (3w). According to the general procedure, menadione 1a and 2chloro-5-nitrobenzyl bromide $\mathbf{2 w}$ were used. After chromatography on silica gel (Cyclohexane:Toluene $=4: 6, \mathrm{UV}), 119 \mathrm{mg}(0.35$ mmol, 70\% yield) of $\mathbf{3 w}$ were isolated as a yellow solid. m.p: 172 $173^{\circ} \mathrm{C}^{1}{ }^{1} \mathrm{H}$ NMR $(400 \mathrm{MHz}$, Chloroform- $\boldsymbol{d}) \boldsymbol{\delta} 8.15-8.12(\mathrm{~m}, 1 \mathrm{H})$, $8.10-8.05(\mathrm{~m}, 1 \mathrm{H}), 8.03(\mathrm{dd}, J=8.8,2.7 \mathrm{~Hz}, 1 \mathrm{H}), 7.85(\mathrm{~d}, J=2.7$ $\mathrm{Hz}, 1 \mathrm{H}), 7.79-7.68(\mathrm{~m}, 2 \mathrm{H}), 7.57(\mathrm{~d}, J=8.7 \mathrm{~Hz}, 1 \mathrm{H}), 4.17(\mathrm{~s}, 2 \mathrm{H})$, 2.19 (s, 3H). ${ }^{13} \mathbf{C}\left\{{ }^{1} \mathbf{H}\right\}$ NMR (101 MHz, Chloroform-d) $\boldsymbol{\delta} 184.8$, $184.3,146.9,146.6,142.8,141.1,138.0,134.0,134.1,132.2$, 131.9, 130.6, 126.8, 126.7, 124.0, 122.9, 30.5, 13.6. HRMS (ESI) $\mathbf{m} / \mathbf{z}:[\mathrm{M}+\mathrm{H}]^{+}$Calcd for $\mathrm{C}_{18} \mathrm{H}_{13} \mathrm{ClNO}_{4}$ 342.0528; Found 342.0529 .

2-(2,5-dibromobenzyl)-3-methylnaphthalene-1,4-dione (3x). According to the general procedure, menadione 1a and 2,5-dibromobenzyl bromide $\mathbf{2 x}$ were used. After chromatography on silica gel (Cyclohexane:Toluene = 4:6, UV), $156 \mathrm{mg}(0.37 \mathrm{mmol}, 74 \%$ yield) of $3 \mathbf{x}$ were isolated as a yellow solid. m.p: $126-127^{\circ} \mathbf{C} .{ }^{1} \mathbf{H}$ NMR (400 MHz, Chloroform-d) $\delta$ 8.22 - 8.02 (m, 2H), $7.83-$ $7.67(\mathrm{~m}, 2 \mathrm{H}), 7.45(\mathrm{~d}, J=8.5 \mathrm{~Hz}, 1 \mathrm{H}), 7.20(\mathrm{dd}, J=8.5,2.4 \mathrm{~Hz}$, $1 \mathrm{H}), 7.00(\mathrm{~d}, J=2.3 \mathrm{~Hz}, 1 \mathrm{H}), 4.09$ (s, 2H), 2.14 (s, 3H). ${ }^{13} \mathbf{C}\left\{{ }^{1} \mathbf{H}\right\}$ NMR (101 MHz, Chloroform-d) $\delta$ 185.0, 184.4, 146.4, 143.9, $139.8,134.4,133.9,132.3,132.1,131.6,131.3,126.8,126.6$, 123.5, 121.8, 32.8, 13.6. HRMS (ESI) $\mathbf{m} / \mathbf{z}:[\mathrm{M}+\mathrm{H}]^{+}$Calcd for $\mathrm{C}_{18} \mathrm{H}_{13} \mathrm{Br}_{2} \mathrm{O}_{2}$ 418.9277; Found 418.9279.

2-(4-bromo-2-fluorobenzyl)-3-methylnaphthalene-1,4-dione (3y). According to the general procedure, menadione 1a and 4bromo-2-fluorobenzyl bromide $\mathbf{2 y}$ were used. After chromatography on silica gel (Cyclohexane:Toluene $=4: 6, \mathrm{UV}), 129 \mathrm{mg}(0.36$ mmol, 72\% yield) of $\mathbf{3 y}$ were isolated as a yellow solid. m.p: $108-$ $109^{\circ} \mathrm{C}^{1}{ }^{\mathbf{H}} \mathrm{H}$ NMR (500 MHz, Chloroform- $\left.\boldsymbol{d}\right) \boldsymbol{\delta} 8.13-8.00(\mathrm{~m}, 2 \mathrm{H})$, $7.81-7.58(\mathrm{~m}, 2 \mathrm{H}), 7.21(\mathrm{dd}, J=9.6,2.0 \mathrm{~Hz}, 1 \mathrm{H}), 7.16(\mathrm{dd}, J=$ $8.3,2.0 \mathrm{~Hz}, 1 \mathrm{H}), 7.06(\mathrm{t}, J=8.1 \mathrm{~Hz}, 1 \mathrm{H}), 3.98(\mathrm{~s}, 2 \mathrm{H}), 2.21(\mathrm{~s}, 3 \mathrm{H})$. ${ }^{19}$ F NMR (471 MHz, Chloroform- $d$ ) $\delta$-113.57 (t, $J=8.7 \mathrm{~Hz}$ ). ${ }^{13} \mathbf{C}\left\{{ }^{1} \mathbf{H}\right\}$ NMR (126 MHz, Chloroform-d) $\delta$ 185.2, 184.6, 160.7 $(\mathrm{d}, J=250.3 \mathrm{~Hz}), 145.5,143.8,133.8,132.2,132.1,131.7$ (d, $J=$ $4.9 \mathrm{~Hz}), 127.7(\mathrm{~d}, J=3.6 \mathrm{~Hz}), 126.6,126.5,124.3(\mathrm{~d}, J=15.7 \mathrm{~Hz})$, 120.5 (d, $J=9.6 \mathrm{~Hz}), 119.2$ (d, $J=25.6 \mathrm{~Hz}), 25.5$ (d, $J=3.1 \mathrm{~Hz})$, $13.2\left(\mathrm{~d}, J=2.7 \mathrm{~Hz}\right.$ ). HRMS (ESI) $\mathbf{m} / \mathbf{z}:[\mathrm{M}+\mathrm{H}]^{+}$Calcd for $\mathrm{C}_{18} \mathrm{H}_{13} \mathrm{BrFO}_{2}$ 359.0077; Found 359.0065.

2-(3-fluoro-4-(trifluoromethyl)benzyl)-3-methylnaphthalene1,4-dione (3z). According to the general procedure, menadione 1a and 3-fluro-4-trifluoromethylbenzyl bromide $\mathbf{2 z}$ were used. After chromatography on silica gel (Cyclohexane:Toluene $=4: 6$, UV), $129 \mathrm{mg}$ ( $0.37 \mathrm{mmol}, 74 \%$ yield) of $\mathbf{3 z}$ were isolated as a yellow solid. m.p: $85-86{ }^{\circ} \mathrm{C} .{ }^{\mathbf{1}} \mathbf{H}$ NMR (500 MHz, Chloroform-d) $\delta$ 8.16 $-8.00(\mathrm{~m}, 2 \mathrm{H}), 7.81-7.66(\mathrm{~m}, 2 \mathrm{H}), 7.50(\mathrm{t}, J=7.7 \mathrm{~Hz}, 1 \mathrm{H}), 7.12$ (dd, $J=8.1,1.2 \mathrm{~Hz}, 1 \mathrm{H}), 7.09-7.02(\mathrm{~m}, 1 \mathrm{H}), 4.07$ (s, 2H), 2.25 (s, 3H). ${ }^{19}$ F NMR (471 MHz, Chloroform-d) $\delta-61.20$ (d, $J=12.2$ $\mathrm{Hz}),-114.07(\mathrm{td}, J=12.0,7.3 \mathrm{~Hz}) .{ }^{13} \mathbf{C}\left\{{ }^{1} \mathbf{H}\right\}$ NMR $(\mathbf{1 2 6} \mathbf{~ M H z}$, Chloroform-d) $\boldsymbol{\delta} 185.1,184.5,160.1(\mathrm{dq}, J=256.4,2.1 \mathrm{~Hz})$, $145.4,145.3,143.8,133.93,133.88,132.2,131.9,127.5(\mathrm{qd}, J=$ 4.4, $2.1 \mathrm{~Hz}), 126.7,126.6,124.3(\mathrm{~d}, J=3.5 \mathrm{~Hz}), 122.7$ (q, $J=272.0$ $\mathrm{Hz}), 117.1(\mathrm{~d}, J=21.0 \mathrm{~Hz}), 116.7(\mathrm{qd}, J=33.2,12.3 \mathrm{~Hz}), 32.4$, 
13.5. HRMS (ESI) m/z: $[\mathrm{M}+\mathrm{H}]^{+}$Calcd for $\mathrm{C}_{19} \mathrm{H}_{13} \mathrm{~F}_{4} \mathrm{O}_{2} 349.0846$; Found 349.0836.

2-methyl-3-((perfluorophenyl)methyl)naphthalene-1,4-dione (3aa). According to the general procedure, menadione 1a and 2,3,4,5,6-pentafluorobenzyl bromide 2aa were used. After chromatography on silica gel (Cyclohexane:Toluene $=4: 6, \mathrm{UV}), 125 \mathrm{mg}$ ( $0.35 \mathrm{mmol}, 71 \%$ yield) of 3aa were isolated as a yellow solid. m.p: 103-104 ${ }^{\circ} \mathrm{C}$. ${ }^{1} \mathbf{H}$ NMR (400 MHz, Chloroform-d) $\delta 8.09-8.04$ (m, 1H), $8.03-7.98(\mathrm{~m}, 1 \mathrm{H}), 7.75-7.63(\mathrm{~m}, 2 \mathrm{H}), 4.03(\mathrm{~s}, 2 \mathrm{H})$, 2.24 (s, 3H). ${ }^{19}$ F NMR (471 MHz, Chloroform- $d$ ) $\delta$-141.23 - $141.33(\mathrm{~m}),-156.45$ (t, $J=20.9 \mathrm{~Hz}),-162.22--162.38(\mathrm{~m})$. ${ }^{13} \mathbf{C}\left\{{ }^{1} \mathbf{H}\right\}$ NMR (126 MHz, Chloroform- $d$ ) $\boldsymbol{\delta}$ 184.8, 183.8, 145.5, $145.4(\mathrm{dm}, J=247.0 \mathrm{~Hz}), 142.0,140.2(\mathrm{dm}, J=252.7 \mathrm{~Hz}), 138.8$ $-136.4(\mathrm{dm}, J=251.8 \mathrm{~Hz}), 133.83,133.80,132.1,131.9,126.57$, 126.56, 112.1 (td, $J=17.5,4.1 \mathrm{~Hz}), 21.0,13.0$. HRMS (ESI) m/z: $[\mathrm{M}+\mathrm{H}]^{+}$Calcd for $\mathrm{C}_{18} \mathrm{H}_{10} \mathrm{~F}_{5} \mathrm{O}_{2}$ 353.0595; Found 353.0573 .

2-methyl-3-(naphthalen-2-ylmethyl)naphthalene-1,4-dione (3ab). According to the general procedure, menadione 1a and 2(bromomethyl)naphthalene 2ab were used. After chromatography on silica gel (Cyclohexane:Toluene $=4: 6$, UV), $107 \mathrm{mg}(0.34$ mmol, 69\% yield) of 3ab were isolated as a yellow solid. m.p: 109$110{ }^{\circ} \mathrm{C} .{ }^{1} \mathbf{H}$ NMR (400 MHz, Chloroform-d) $\delta 8.20-8.04(\mathrm{~m}$, $2 \mathrm{H}), 7.85-7.67(\mathrm{~m}, 5 \mathrm{H}), 7.64-7.60(\mathrm{~m}, 1 \mathrm{H}), 7.51-7.34(\mathrm{~m}, 3 \mathrm{H})$, 4.21 (s, 2H), 2.29 (s, 3H). ${ }^{13} \mathbf{C}\left\{{ }^{1} \mathbf{H}\right\}$ NMR (101 MHz, Chloroformd) $\delta 185.6,184.9,145.4,144.8,135.7,133.68,133.65,132.32$, $132.30,132.2,128.5,127.7,127.7,127.3,126.9,126.7,126.5$, 126.3, 125.7, 32.7, 13.5. HRMS (ESI) $\mathbf{m} / \mathbf{z}:[\mathrm{M}+\mathrm{K}]^{+}$Calcd for $\mathrm{C}_{22} \mathrm{H}_{16} \mathrm{KO}_{2}$ 351.0782; Found 351.0806.

2-allyl-3-methylnaphthalene-1,4-dione (3ac). A variant from the general procedure was performed. In a $10 \mathrm{~mL}$ tube, menadione $\mathbf{1 a}$ $(0.5 \mathrm{mmol})$, allyl bromide $2 \mathrm{ac}(1 \mathrm{mmol})$ and $\mathrm{Fe}(\mathrm{acac})_{3}(10 \%)$ were dissolved in Acetonitrile ( $5 \mathrm{~mL}$ ). The mixture was stirred and 2,6lutidine $(0.6 \mathrm{mmol})$ and $\gamma$-terpinene $(0.6 \mathrm{mmol})$ were added successively. The tube was sealed, placed under blue light irradiation and heated up at $90^{\circ} \mathrm{C}$ during $24 \mathrm{~h}$. After completion, the mixture was allowed to cool down at room temperature and was partitioned between ethyl acetate $(10 \mathrm{~mL})$ and aqueous $1 \mathrm{M} \mathrm{HCl}(10 \mathrm{~mL})$. The aqueous layer was extracted once with $10 \mathrm{~mL}$ ethyl acetate and the reunited organic layers were washed with brine and dried with $\mathrm{MgSO}_{4}$. The solvent was removed and the crude was purified by silica gel chromatography (Cyclohexane:Ethyl Acetate $=95: 5, \mathrm{UV})$ to obtained $72 \mathrm{mg}(0.34 \mathrm{mmol}, 68 \%)$ of $\mathbf{3 a c}$ as an orange solid. m.p: $67-68^{\circ} \mathrm{C} .{ }^{1} \mathbf{H}$ NMR (500 MHz, Chloroform-d) $\delta$ 8 $8.11-8.03$ $(\mathrm{m}, 2 \mathrm{H}), 7.75-7.60(\mathrm{~m}, 2 \mathrm{H}), 5.83$ (ddt, $J=17.1,10.1,6.3 \mathrm{~Hz}, 1 \mathrm{H})$, $5.15-5.01(\mathrm{~m}, 2 \mathrm{H}), 3.41(\mathrm{dd}, J=6.3,0.7 \mathrm{~Hz}, 2 \mathrm{H}), 2.19$ (s, 3H). ${ }^{13} \mathbf{C}\left\{{ }^{1} \mathbf{H}\right\}$ NMR (126 MHz, Chloroform- $d$ ) $\delta$ 185.4, 184.4, 144.41, 144.40, 133.6, 133.5, 133.32, 132.27, 132.2, 126.5, 126.4, 116.8, 31.1, 12.7. HRMS (ESI) $\mathbf{m} / \mathbf{z}:[\mathrm{M}+\mathrm{H}]^{+}$Calcd for $\mathrm{C}_{14} \mathrm{H}_{13} \mathrm{O}_{2}$ 213.0910; Found 213.0909.

2-chloro-3-(4-(trifluoromethyl)benzyl)naphthalene-1,4-dione (5a). According to the general procedure, 2-chloronaphthalene-1,4dione $\mathbf{4 a}$ and 4-trifluoromethylbenzyl bromide $\mathbf{2} \mathbf{a}$ were used. After chromatography on silica gel (Cyclohexane:Toluene $=4: 6$, UV), $95 \mathrm{mg}(0.27 \mathrm{mmol}, 54 \%$ yield $)$ of $\mathbf{5 a}$ were isolated as a yellow solid. m.p: $160-161{ }^{\circ} \mathrm{C}^{\mathbf{1}}{ }^{\mathrm{H}}$ NMR (500 MHz, Chloroform-d) $\delta$ $8.19-$ $8.15(\mathrm{~m}, 1 \mathrm{H}), 8.11(\mathrm{~m}, 1 \mathrm{H}), 7.81-7.72(\mathrm{~m}, 2 \mathrm{H}), 7.54(\mathrm{~d}, J=8.4$ $\mathrm{Hz}, 2 \mathrm{H}), 7.49$ (d, $J=8.3 \mathrm{~Hz}, 2 \mathrm{H}), 4.23(\mathrm{~s}, 3 \mathrm{H}) .{ }^{19} \mathbf{F}$ NMR $(471$ MHz, Chloroform- $d$ ) $-62.54 .{ }^{13} \mathbf{C}\left\{{ }^{1} \mathbf{H}\right\}$ NMR (126 MHz, Chloroform-d) $\delta 182.4,177.8,145.7,144.4,140.9,134.6,134.4,131.6$, $131.4,129.7,129.4(\mathrm{q}, J=32.6 \mathrm{~Hz}), 127.5,127.4,125.8(\mathrm{q}, J=3.7$ $\mathrm{Hz}$ ), 124.2 (q, $J=272.3 \mathrm{~Hz}$ ), 33.9. HRMS (ESI) m/z: $[\mathrm{M}+\mathrm{H}]^{+}$ Calcd for $\mathrm{C}_{18} \mathrm{H}_{11} \mathrm{ClF}_{3} \mathrm{O}_{2}$ 351.0394; Found 351.0403. 2-bromo-3-(4-(trifluoromethyl)benzyl)naphthalene-1,4-dione (5b). According to the general procedure, 2-bromonaphthalene1,4-dione $\mathbf{4 b}$ and 4-trifluoromethylbenzyl bromide $\mathbf{2 a}$ were used. After chromatography on silica gel (Cyclohexane:Toluene $=4: 6$, UV), $84 \mathrm{mg}(0.21 \mathrm{mmol}, 43 \%$ yield $)$ of $\mathbf{5 b}$ were isolated as a yellow solid. m.p: $126-127^{\circ} \mathrm{C} .{ }^{\mathbf{1}} \mathbf{H}$ NMR (400 MHz, Chloroform-d) $\delta$ $8.19-8.15(\mathrm{~m}, 1 \mathrm{H}), 8.14-8.08(\mathrm{~m}, 1 \mathrm{H}), 7.85-7.70(\mathrm{~m}, 2 \mathrm{H}), 7.59$ $-7.48(\mathrm{~m}, 4 \mathrm{H}), 4.29$ (s, 2H). ${ }^{19} \mathbf{F}$ NMR (377 MHz, Chloroform-d) $\boldsymbol{\delta}-62.56 .{ }^{13} \mathbf{C}\left\{{ }^{1} \mathbf{H}\right\}$ NMR (101 MHz, Chloroform-d) $\boldsymbol{\delta} 181.8$, $177.8,149.2,140.8,140.5,134.5,134.3,131.5,131.2,129.6,129.1$ $(\mathrm{q}, J=36.5 \mathrm{~Hz}), 127.8,127.5,125.7$ (q, $J=3.8 \mathrm{~Hz}), 124.2$ (q, $J=$ $272.0 \mathrm{~Hz}$ ), 36.9. HRMS (ESI) $\mathbf{m} / \mathbf{z}: \quad[\mathrm{M}+\mathrm{H}]^{+}$Calcd for $\mathrm{C}_{18} \mathrm{H}_{11} \mathrm{BrF}_{3} \mathrm{O}_{2}$ 394.9889. Found 394.9886.

2-phenyl-3-(4-(trifluoromethyl)benzyl)naphthalene-1,4-dione (5c). According to the general procedure, 2-phenylnaphthalene1,4-dione $\mathbf{4 c}$ and 4-trifluoromethylbenzyl bromide $\mathbf{2 a}$ were used. After chromatography on silica gel (Cyclohexane:Toluene $=1: 1$, UV), $175 \mathrm{mg}$ ( $0.45 \mathrm{mmol}, 89 \%$ yield) of $\mathbf{5 c}$ were isolated as an orange solid. m.p: $93-94{ }^{\circ} \mathrm{C}^{\mathbf{1}} \mathbf{H}$ NMR (500 MHz, Chloroform-d) 反 $8.22-8.04(\mathrm{~m}, 2 \mathrm{H}), 7.76(\mathrm{~m}, 2 \mathrm{H}), 7.55-7.39(\mathrm{~m}, 5 \mathrm{H}), 7.25-$ $7.16(\mathrm{~m}, 2 \mathrm{H}), 7.14-7.06(\mathrm{~m}, 2 \mathrm{H}), 3.97(\mathrm{~s}, 2 \mathrm{H}) .{ }^{19} \mathbf{F}$ NMR $(471$ MHz, Chloroform- $d$ ) $\delta$-62.44. ${ }^{13} \mathbf{C}\left\{{ }^{1} \mathbf{H}\right\}$ NMR (126 MHz, Chloroform- $\boldsymbol{d}) \boldsymbol{\delta} 185.3,184.6,147.7,145.1,142.8,134.1,134.0,133.2$, $132.1,129.2,129.1,129.0,128.8(\mathrm{q}, J=32.4 \mathrm{~Hz}), 128.6,126.9$, 126.7, 125.5 (q, $J=3.8 \mathrm{~Hz}), 124.3$ (q, $J=271.8 \mathrm{~Hz}), 33.4$. HRMS (ESI) $\mathbf{m} / \mathbf{z}:[\mathrm{M}+\mathrm{H}]^{+}$Calcd for $\mathrm{C}_{24} \mathrm{H}_{16} \mathrm{~F}_{3} \mathrm{O}_{2}:$ 393.1097; Found 393.1117.

2-ethyl-3-(4-(trifluoromethyl)benzyl)naphthalene-1,4-dione (5d). According to the general procedure, 2-ethylnaphthalene-1,4dione 4d and 4-trifluoromethylbenzyl bromide $\mathbf{2 a}$ were used. After chromatography on silica gel (Cyclohexane:Toluene $=1: 1, \mathrm{UV}$ ), $108 \mathrm{mg}(0.31 \mathrm{mmol}, 63 \%$ yield) of $\mathbf{5 d}$ were isolated as an orange solid. m.p: $77-78{ }^{\circ} \mathrm{C} .{ }^{1} \mathbf{H}$ NMR (500 MHz, Chloroform- $d$ ) $\delta$ 8.15 $-7.99(\mathrm{~m}, 2 \mathrm{H}), 7.78-7.65(\mathrm{~m}, 2 \mathrm{H}), 7.52(\mathrm{~d}, J=8.0 \mathrm{~Hz}, 2 \mathrm{H}), 7.34$ $(\mathrm{d}, J=8.0 \mathrm{~Hz}, 2 \mathrm{H}), 4.09$ (s, 2H), $2.72(\mathrm{q}, J=7.6 \mathrm{~Hz}, 2 \mathrm{H}), 1.09$ (t, $J=7.5 \mathrm{~Hz}, 3 \mathrm{H}) .{ }^{19} \mathbf{F}$ NMR (471 MHz, Chloroform- $d$ ) $\delta$-62.44. ${ }^{13} \mathbf{C}\left\{{ }^{1} \mathbf{H}\right\}$ NMR (126 MHz, Chloroform-d) $\delta$ 185.1, 184.9, 150.2, $143.8,142.8,133.80,133.75,132.4,132.1,128.9(\mathrm{q}, J=32.4 \mathrm{~Hz})$, $128.9,126.6,126.5,125.7(\mathrm{q}, J=3.7 \mathrm{~Hz}), 124.3(\mathrm{q}, J=271.9 \mathrm{~Hz})$, 32.1, 21.1, 13.6. HRMS (ESI) $\mathbf{m} / \mathbf{z}:[\mathrm{M}+\mathrm{H}]^{+}$Calcd for $\mathrm{C}_{20} \mathrm{H}_{16} \mathrm{~F}_{3} \mathrm{O}_{2}$ 345.1097; Found 345.1099.

2-benzyl-3-(4-(trifluoromethyl)benzyl)naphthalene-1,4-dione (5e). According to the general procedure, 2-benzylnaphthalene1,4-dione 4e and 4-trifluoromethylbenzyl bromide $\mathbf{2 a}$ were used. After chromatography on silica gel (Cyclohexane:Toluene $=4: 6$, $\mathrm{UV}), 113 \mathrm{mg}(0.28 \mathrm{mmol}, 56 \%$ yield) of $\mathbf{5 e}$ were isolated as a yellow solid. m.p: $93-94{ }^{\circ} \mathrm{C} .{ }^{\mathbf{1}} \mathbf{H}$ NMR (500 MHz, Chloroform-d) $8.17-8.01(\mathrm{~m}, 2 \mathrm{H}), 7.83-7.66(\mathrm{~m}, 2 \mathrm{H}), 7.48(\mathrm{~d}, J=8.1 \mathrm{~Hz}, 2 \mathrm{H})$, $7.27-7.12(\mathrm{~m}, 7 \mathrm{H}), 4.13$ (s, 2H), 4.10 (s, 2H). ${ }^{19}$ F NMR (471 MHz, Chloroform- $d$ ) $\delta$ - $62.45 .{ }^{13} \mathbf{C}\left\{{ }^{1} \mathbf{H}\right\}$ NMR (126 MHz, Chloroform-d) $\boldsymbol{\delta} 185.2,185.1,146.8,145.5,142.2,137.8,134.0,133.9$, $132.1,132.1,128.97,128.96(\mathrm{q}, J=32.6 \mathrm{~Hz}), 128.9,128.6,126.8$, $126.7,125.7(\mathrm{q}, J=3.9 \mathrm{~Hz}), 124.3(\mathrm{q}, J=272.1 \mathrm{~Hz}), 32.63,32.61$ HRMS (ESI) $\mathbf{m} / \mathbf{z}:[\mathrm{M}+\mathrm{H}]^{+}$Calcd for $\mathrm{C}_{25} \mathrm{H}_{18} \mathrm{~F}_{3} \mathrm{O}_{2} 407.1253$. Found 407.1286.

2-phenoxy-3-(4-(trifluoromethyl)benzyl)naphthalene-1,4-dione (5f). According to the general procedure, 2-phenoxynaphthalene-1,4-dione $\mathbf{4 f}$ and 4-trifluoromethylbenzyl bromide $\mathbf{2 a}$ were used. After chromatography on silica gel (Cyclohexane:Toluene = $1: 1, \mathrm{UV}), 85 \mathrm{mg}(0.21 \mathrm{mmol}, 42 \%$ yield $)$ of $\mathbf{5 f}$ were isolated as a yellow solid. m.p: $156-157^{\circ} \mathrm{C} .{ }^{1} \mathbf{H}$ NMR $(500 \mathrm{MHz}$, Chloroformd) $\delta 8.22-8.11(\mathrm{~m}, 1 \mathrm{H}), 8.02-7.94(\mathrm{~m}, 1 \mathrm{H}), 7.75(\mathrm{td}, J=7.5,1.5$ $\mathrm{Hz}, 1 \mathrm{H}), 7.71(\mathrm{td}, J=7.5,1.5 \mathrm{~Hz}, 1 \mathrm{H}), 7.52-7.43(\mathrm{~m}, 4 \mathrm{H}), 7.35-$ 
$7.29(\mathrm{~m}, 2 \mathrm{H}), 7.15-7.08(\mathrm{~m}, 1 \mathrm{H}), 6.94-6.88(\mathrm{~m}, 2 \mathrm{H}), 4.08(\mathrm{~s}$, 2H). ${ }^{19} \mathbf{F}$ NMR (471 MHz, Chloroform-d) $\delta-62.44 .{ }^{13} \mathbf{C}\left\{{ }^{1} \mathbf{H}\right\}$ NMR (126 MHz, Chloroform-d) $\delta$ 184.9, 179.8, 157.1, 154.0, $142.2,136.7,134.4,134.1,132.0,131.3,129.9,129.8,129.0$ (q, $J$ $=32.4 \mathrm{~Hz}), 126.8,126.8,125.6(\mathrm{q}, J=3.8 \mathrm{~Hz}), 124.3(\mathrm{q}, J=272.0$ $\mathrm{Hz}$ ), 123.7, 116.4, 29.8. HRMS (ESI) $\mathbf{m} / \mathbf{z}:[\mathrm{M}+\mathrm{H}]^{+}$Calcd for $\mathrm{C}_{24} \mathrm{H}_{16} \mathrm{~F}_{3} \mathrm{O}_{3}$ 409.1046; Found 409.1030.

1,4-dioxo-3-(4-(trifluoromethyl)benzyl)-1,4-dihydronaphthalen-2-yl acetate $(5 \mathrm{~g})$. According to the general procedure, 1,4-dioxo-1,4-dihydronaphthalen-2-yl acetate $\mathbf{4 g}$ and 4-trifluoromethylbenzyl bromide 2a were used. After chromatography on silica gel (Cyclohexane:Toluene = 1:9, UV), $91 \mathrm{mg}(0.24 \mathrm{mmol}, 49 \%$ yield) of $\mathbf{5 g}$ were isolated as an orange solid. m.p: $120-121{ }^{\circ} \mathbf{C} .{ }^{\mathbf{1}} \mathbf{H}$ NMR (500 MHz, Chloroform-d) $\delta 8.16-8.04$ (m, 2H), 7.77 $7.70(\mathrm{~m}, 2 \mathrm{H}), 7.53(\mathrm{~d}, J=8.1 \mathrm{~Hz}, 2 \mathrm{H}), 7.41(\mathrm{~d}, J=8.4 \mathrm{~Hz}, 2 \mathrm{H})$, 3.97 (s, 2H), 2.41 (s, 3H). ${ }^{19}$ F NMR (471 MHz, Chloroform-d) $\delta$ -62.49. ${ }^{13} \mathbf{C}\left\{{ }^{1} \mathbf{H}\right\}$ NMR (126 MHz, Chloroform-d) $\boldsymbol{\delta}$ 184.2, 178.2, $167.9,151.9,141.4,136.8,134.5,134.2,131.9,130.9,129.4,129.2$ $(\mathrm{q}, J=32.4 \mathrm{~Hz}), 127.0,126.9,125.7(\mathrm{q}, J=3.8 \mathrm{~Hz}), 124.2(\mathrm{q}, J=$ $272.0 \mathrm{~Hz}$ ), 30.1, 20.5. HRMS (ESI) $\mathbf{m} / \mathbf{z}:[\mathrm{M}+\mathrm{Na}]^{+}$Calcd for $\mathrm{C}_{20} \mathrm{H}_{13} \mathrm{~F}_{3} \mathrm{NaO}_{4}$ 397.0658 Found 397.0658.

5-hydroxy-2-methyl-3-(4-(trifluoromethyl)benzyl)naphthalene-1,4-dione $(5 \mathrm{~h})$. According to the general procedure, plumbagin $\mathbf{4 h}$ and 4-trifluoromethylbenzyl bromide $\mathbf{2 a}$ were used. After chromatography on silica gel (Cyclohexane:Toluene $=4: 6$, UV), $64 \mathrm{mg}(0.185 \mathrm{mmol}, 37 \%$ yield $)$ of $\mathbf{5 h}$ were isolated as an orange

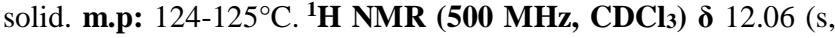
$1 \mathrm{H}), 7.64-7.49(\mathrm{~m}, 4 \mathrm{H}), 7.35(\mathrm{~d}, J=8.0 \mathrm{~Hz}, 2 \mathrm{H}), 7.22(\mathrm{dd}, J=$ 8.2, 1.3 Hz, 1H), 4.07 (s, 2H), $2.24(\mathrm{~s}, 3 \mathrm{H}) .{ }^{19}$ F NMR (471 MHz, $\left.\mathbf{C D C l}_{3}\right) \delta$-62.46. ${ }^{13} \mathbf{C}\left\{{ }^{1} \mathbf{H}\right\}$ NMR (126 $\left.\mathbf{M H z}, \mathbf{C D C l}_{3}\right) \boldsymbol{\delta}$ 189.7, $184.4,161.5,146.4,144.3,142.0,136.4,132.2,129.1$ (q, $J=32.4$ $\mathrm{Hz}), 128.9,125.8(\mathrm{q}, J=3.8 \mathrm{~Hz}), 124.23,124.22(\mathrm{q}, J=272.1 \mathrm{~Hz})$, 119.3, 114.9, 31.9, 13.6. HRMS (ESI) $\mathbf{m} / \mathbf{z}:[\mathrm{M}+\mathrm{H}]^{+}$Calcd for $\mathrm{C}_{19} \mathrm{H}_{14} \mathrm{~F}_{3} \mathrm{O}_{3}$ 347.0890; Found 347.0910.

5-methoxy-2-methyl-3-(4-(trifluoromethyl)benzyl)naphthalene-1,4-dione (5i). According to the general procedure, 5-methoxy-2-methylnaphthalene-1,4-dione $\mathbf{4} \mathbf{i}$ and 4-trifluoromethylbenzyl bromide 2a were used. After chromatography on silica gel (Cyclohexane:Toluene $=4: 6, \mathrm{UV}), 90 \mathrm{mg}(0.25 \mathrm{mmol}, 50 \%$ yield $)$ of $\mathbf{5 i}$ were isolated as an orange solid. m.p: $136-137^{\circ} \mathrm{C} .{ }^{\mathbf{1}} \mathbf{H}$ NMR (400 MHz, Chloroform- $\boldsymbol{d}$ ) $\boldsymbol{\delta} 7.76(\mathrm{dd}, J=7.6,1.1 \mathrm{~Hz}, 1 \mathrm{H}), 7.64(\mathrm{dd}, J$ $=8.5,7.7 \mathrm{~Hz}, 1 \mathrm{H}), 7.50(\mathrm{~d}, J=8.1 \mathrm{~Hz}, 2 \mathrm{H}), 7.35(\mathrm{~d}, J=7.9 \mathrm{~Hz}$, 2H), 7.27 (d, $J=8.5 \mathrm{~Hz}, 1 \mathrm{H}), 4.05$ (s, 2H), 3.99 (s, 3H), 2.19 (s, 3H). ${ }^{19}$ F NMR (377 MHz, Chloroform-d) $\delta-62.45 .{ }^{13} \mathbf{C}\left\{{ }^{1} \mathbf{H}\right\}$ NMR (101 MHz, Chloroform-d) $\delta$ 185.5, 183.8, 159.7, 146.2, 142.7, 142.6, 134.9, 134.4, 129.1, 128.8 (q, $J=32.5 \mathrm{~Hz}), 125.6(\mathrm{q}$, $J=3.6 \mathrm{~Hz}), 124.3(\mathrm{q}, J=271.8 \mathrm{~Hz}), 119.8,119.3,117.7,56.6,32.7$, 13.2. HRMS (ESI) m/z: $[\mathrm{M}+\mathrm{K}]^{+}$Calcd for $\mathrm{C}_{20} \mathrm{H}_{15} \mathrm{~F}_{3} \mathrm{KO}_{3} 399.0605$ Found 399.0635.

6-fluoro-2-methyl-3-(4-(trifluoromethyl)benzyl)naphthalene1,4-dione (5j). According to the general procedure, 6-fluoro-2-methylnaphthalene-1,4-dione $\mathbf{4 j}$ and 4-trifluoromethylbenzyl bromide 2a were used. After chromatography on silica gel (Cyclohexane:Toluene $=1: 1, \mathrm{UV}), 116 \mathrm{mg}(0.33 \mathrm{mmol}, 67 \%$ yield $)$ of $\mathbf{5 j}$ were isolated as a yellow solid. m.p: $105-105^{\circ} \mathrm{C} .{ }^{1} \mathbf{H}$ NMR $(500 \mathrm{MHz}$, Chloroform- $\boldsymbol{d}$ ) $\boldsymbol{\delta} 8.13(\mathrm{dd}, J=8.6,5.2 \mathrm{~Hz}, 1 \mathrm{H}), 7.72$ (dd, $J=8.5$, $2.7 \mathrm{~Hz}, 1 \mathrm{H}), 7.55-7.48(\mathrm{~m}, 2 \mathrm{H}), 7.40-7.30(\mathrm{~m}, 3 \mathrm{H}), 4.08(\mathrm{~s}, 2 \mathrm{H})$, $2.26(\mathrm{~s}, 3 \mathrm{H}) .{ }^{19} \mathbf{F}$ NMR (471 MHz, Chloroform-d) $\delta$-62.49, 102.18 (td, $J=8.4,5.3 \mathrm{~Hz}) .{ }^{13} \mathbf{C}\left\{{ }^{1} \mathbf{H}\right\}$ NMR $(\mathbf{1 2 6}$ MHz, Chloroform- $d) \delta 183.8,183.5,166.1(\mathrm{~d}, J=257.1 \mathrm{~Hz}), 145.2,144.6(\mathrm{~d}, J$ $=1.8 \mathrm{~Hz}), 142.0,134.5(\mathrm{~d}, J=7.8 \mathrm{~Hz}), 129.7(\mathrm{~d}, J=8.9 \mathrm{~Hz}), 129.0$ $(\mathrm{q}, J=32.3 \mathrm{~Hz}), 128.9,128.7(\mathrm{~d}, J=3.3 \mathrm{~Hz}), 125.7(\mathrm{q}, J=3.8 \mathrm{~Hz})$, $124.1(\mathrm{q}, J=271.9 \mathrm{~Hz}), 120.9(\mathrm{~d}, J=22.5 \mathrm{~Hz}), 113.3(\mathrm{~d}, J=23.5$
$\mathrm{Hz}$ ), 32.4, 13.4. HRMS (ESI) $\mathbf{m} / \mathbf{z}:[\mathrm{M}+\mathrm{H}]^{+}$Calcd for $\mathrm{C}_{19} \mathrm{H}_{13} \mathrm{~F}_{4} \mathrm{O}_{2}$ 349.0846 Found 349.0835.

2,3,5-trimethyl-6-(4-(trifluoromethyl)benzyl)cyclohexa-2,5diene-1,4-dione (5k). According to the general procedure, 2,3,5trimethylbenzoquinone $\mathbf{4 k}$ and 4-trifluoromethylbenzyl bromide 2a were used. After chromatography on silica gel (Cyclohexane:Toluene $=4: 6, \mathrm{UV}), 71 \mathrm{mg}(0.23 \mathrm{mmol}, 46 \%$ yield $)$ of $\mathbf{5 k}$ were isolated as a yellow solid. m.p: $74-75^{\circ} \mathrm{C} .{ }^{1} \mathbf{H}$ NMR $(500 \mathrm{MHz}$, $\left.\mathbf{C D C l}_{3}\right) \boldsymbol{\delta} 7.51(\mathrm{~d}, J=8.1 \mathrm{~Hz}, 2 \mathrm{H}), 7.29(\mathrm{~d}, J=8.0 \mathrm{~Hz}, 2 \mathrm{H}), 3.91$ $(\mathrm{s}, 2 \mathrm{H}), 2.10(\mathrm{~s}, 3 \mathrm{H}), 2.03(\mathrm{~d}, J=1.4 \mathrm{~Hz}, 3 \mathrm{H}), 2.01(\mathrm{~d}, J=1.5 \mathrm{~Hz}$, 3H). ${ }^{19} \mathbf{F}$ NMR (471 MHz, $\left.\mathbf{C D C l}_{3}\right) \delta-62.45 .{ }^{13} \mathbf{C}\left\{{ }^{1} \mathbf{H}\right\}$ NMR (126 MHz, $\left.\mathbf{C D C l}_{3}\right) \boldsymbol{\delta} 187.7,187.0,142.6,141.9,141.7,141.0,140.6$, $129.0,129.3-128.4(\mathrm{q}, J=32.5 \mathrm{~Hz}), 125.6(\mathrm{q}, J=3.9 \mathrm{~Hz}), 124.3$ (q, $J=271.8 \mathrm{~Hz}$ ), 32.1, 13.0, 12.57, 12.55. HRMS (ESI) m/z: $[\mathrm{M}+\mathrm{H}]^{+}$Calcd for $\mathrm{C}_{17} \mathrm{H}_{16} \mathrm{~F}_{3} \mathrm{O}_{2}$ 309.1097; Found $309.1096\left(\mathrm{MH}^{+}\right)$.

\section{SUPPORTING INFORMATION}

Detailed screenings of the photochemical reactions, studies of the reaction mechanism, spectrophotometric absorption data and ${ }^{1} \mathrm{H}$ and ${ }^{13} \mathrm{C}$ NMR spectra of all compounds are given in the Supporting Information. The Supporting Information is available free of charge on the ACS Publications website.

\section{AUTHOR INFORMATION}

\section{Corresponding Author}

*E-mail: elisabeth.davioud@unistra.fr

Notes

The authors declare no conflict of interest.

\section{ACKNOWLEDGMENT}

The authors wish to thank the ANR-PRC program (grant PlasmoPrim project, E.D.C.), the Laboratoire d'Excellence (LabEx) ParaFrap (grant LabEx ParaFrap ANR-11-LABX-0024, E.D.C.), for funding and creating a proper framework for this scientific research, and for M. D.'s salary.

\section{REFERENCES}

(1) (a) Müller, T.; Johann, L.; Jannack B.; Brückner, M.; Lanfranchi, D. A.; Bauer, D. H.; Sanchez, C.; Yardley, V.; Deregnaucourt, C.; Schrével, J.; Lanzer, M.; Schirmer, R. H.; Davioud-Charvet, E. Glutathione Reductase-Catalyzed Cascade of Redox Reactions To Bioactivate Potent Antimalarial 1,4-Naphthoquinones - A New Strategy to Combat Malarial Parasites. J. Am. Chem. Soc. 2011, 133, 11557-11571. (b) Ehrhardt, K.; Deregnaucourt, C.; Goetz, A.-A.; Tzanova, T.; Pradines, B.; Adjalley, S. H.; Blandin, S.; Bagrel, D.; Lanzer, M.; Davioud-Charvet, E. The Redox Cycler Plasmodione Is a Fast-Acting Antimalarial Lead Compound with Pronounced Activity against Sexual and Early Asexual Blood-Stage Parasites. Antimicrob. Agents Chemother. 2016, 60, 5146-5158. (c) For a recent review on antimalarial application of quinones: Patel, O. P. S.; Beteck, R. M.; Legoabe, L. J. Antimalarial application of quinones: A recent update. Eur. J. Med. Chem. 2021, 210, 113084.

(2) (a) Cesar Rodo, E.; Feng, F.; Jida, M.; Ehrhardt, K.; Bielitza, M.; Boilevin, J.; Lanzer, M.; Williams, D. L., Lanfranchi, D. A., DavioudCharvet, E. A Platform of Regioselective Methodologies to Access Polysubstituted 2-Methyl-1,4-naphthoquinone Derivatives: Scope and Limitations. Eur. J. Org. Chem. 2016, 11, 1982-1993. (b) Cotos, L.; Donzel, M.; Elhabiri, M.; Davioud-Charvet, E. A Mild and Versatile Friedel-Crafts Methodology for the Diversity-Oriented Synthesis of Redox-Active 3-Benzoylmenadiones with Tunable Redox Potentials. Chem. Eur. J. 2020, 26, 3314-3325. (c) Urgin, K.; Jida, M.; Ehrhardt, 
K.; Müller, T.; Lanzer, M.; Maes, L.; Davioud-Charvet, E. Pharmacomodulation of the Antimalarial Plasmodione: Synthesis of Biaryl- and $\mathrm{N}$-Arylalkylamine Analogues, Antimalarial Activities and Physicochemical Properties. Molecules, 2017, 22, 161, 1-32. (d) Lanfranchi, D. A.; Cesar-Rodo, E.; Bertrand, B.; Huang, H-H.; Day, L.; Johann, L.; Elhabiri, M.; Becker, K.; Williams, D. L.; Davioud-Charvet, E. Synthesis and biological evaluation of 1,4-naphthoquinones and quinoline5,8-diones as antimalarial and schistosomicidal agents. Org. Biomol. Chem. 2012, 10, 6375-6387. (e) Feng, L.; Lanfranchi, D. A.; Cotos, L.; Cesar, E.; Ehrhardt, K.; Goetz, A.-A.; Zimmerman, H.; Fenaille, F.; Blandin, S.; Davioud-Charvet, E. Synthesis of plasmodione metabolites and ${ }^{13} \mathrm{C}$-enriched plasmodione as chemical tools for drug metabolism investigation. Org. Biomol. Chem. 2018, 16, 2647 -2665. (f) Bielitza, M.; Belorgey, D.; Ehrhardt, K.; Johann, L.; Lanfranchi, D. A.; Gallo, V.; Schwarzer, E.; Mohring, F.; Jortzik, E.; Williams, D. L.; Becker, K.; Arese, P.; Elhabiri, M.; Davioud-Charvet, E. Antimalarial NADPH-Consuming Redox-Cyclers As Superior Glucose-6-Phosphate Dehydrogenase Deficiency Copycats. Antioxid. Redox Signal. 2015, 22, 1337-1351.

(3) (a) Jacobsen, N.; Torssell, K. Synthesis of Naturally Occurring Quinones. Alkylation with the Silver Ion-Peroxydisulphate-Carboxylic Acid System. Acta Chem. Scand. 1973, 27, 3211-3216. (b) Goldman, J.; Jacobsen, N.; Torssell, K. Syntheses in the Camphor Series. Alkylation of Quinones with Cycloalkyl Radicals. Attempted Syntheses of Lagopodin A and Desoxyhelicobasidin. Acta Chem. Scand. 1974, 28b, 492-500. (c) Anderson, J. M.; Kochi, J. K. Silver(I)-catalyzed oxidative decarboxylation of acids by peroxydisulfate. Role of silver(II). J. Am. Chem. Soc. 1970, 92, 1651-1659. (d) Salmon-Chemin, L.; Lemaire, A.; De Freitas, S.; Deprez, B.; Sergheraert, C.; Davioud-Charvet, E. Parallel synthesis of a library of 1,4-naphthoquinones and automated screening of potential inhibitors of trypanothione reductase from Trypanosoma cruzi. Bioorg. Med. Chem. Lett. 2000, 10, 631-635. (e) Salmon-Chemin, L.; Buisine, E.; Yardley, V; Kohler, S.; Debreu, M. A.; Landry, V.; Sergheraert, C.; Croft, S.; Krauth-Siegel, L.; DavioudCharvet, E. 2- and 3-Substituted 1,4-Naphthoquinone Derivatives as Subversive Substrates of Trypanothione Reductase and Lipoamide Dehydrogenase from Trypanosoma cruzi: Synthesis and Correlation between Redox Cycling Activities and in Vitro Cytotoxicity. J. Med. Chem. 2001, 44, 548-565.

(4) Sutherland, D. R.; Veguillas, M.; Oates, C. L.; Lee, A-L. Metal-, Photocatalyst-, and Light-Free, Late-Stage C-H Alkylation of Heteroarenes and 1,4-Quinones Using Carboxylic Acids. Org. Lett. 2018, $20,6863-6867$.

(5) (a) Galloway, J. D.; Mai, D. N.; Baxter, R. D. Radical Benzylation of Quinones via $\mathrm{C}-\mathrm{H}$ Abstraction. J. Org. Chem. 2019, 84, 12131-12137. (b) Zhou, S-L.; Guo, L-N.; Duan, X.-H. Copper-Catalyzed Regioselective Cross-Dehydrogenative Coupling of Coumarins with Benzylic $\mathrm{C}_{\mathrm{sp} 3}-\mathrm{H}$ Bonds. Eur. J. Org. Chem. 2014, 8094-8100. (c) Dong, Y.; Yang, J.; He, S.; Shi, Z.-C.; Wang, Y.; Zhang, X.-M.; Wang, J.-Y. Metal-free oxidative cross-dehydrogenative coupling of quinones with benzylic $\mathrm{C}\left(\mathrm{sp}^{3}\right)-\mathrm{H}$ bonds. $R S C A d v$. 2019, 9, 27588-27592. (d) $\mathrm{Li}$ D.; Shen, X. Iron-catalyzed regioselective alkylation of 1,4-quinones and coumarins with functionalized alkyl bromides. Org Biomol Chem. 2020, 18, 750-754

(6) Ramesh, N.; Prakash, C.; Sureshbabu, R.; Dhayalan, V.; Mohanakrishnan, A. K. Unusual dimerization of N-protected bromomethylindoles/benzyl bromide with arylmetal halides: generation of indolylmethyl/benzyl radical. Tetrahedron, 2008, 64, 2071-2079.

(7) (a) Hironaka, K.; Fukuzumi, S.; Tanaka, T. Tris(bipyridyl)ruthenium(II)-photosensitized reaction of 1-benzyl-1,4-dihydronicotinamide with benzyl bromide. J. Chem. Soc., Perkin Trans. 2, 1984, 1705 1709. (b) Kern, J.-M.; Sauvage, J.-P. Photoassisted C-C coupling via electron transfer to benzylic halides by a bis(di-imine) copper(I) complex. J. Chem. Soc., Chem. Commun. 1987, 546-548. (c) Shih, H. W.; Vander Wal, M. N.; Grange, R. L.; MacMillan, D. W. Enantioselective $\alpha$-Benzylation of Aldehydes via Photoredox Organocatalysis. J. Am. Chem. Soc. 2010, 132, 13600-13603. (d) Arceo, E.; Jurberg, I. D.; Alvarez-Fernández, A.; Melchiorre, P. Photochemical activity of a key donor-acceptor complex can drive stereoselective catalytic $\alpha$-alkylation of aldehydes. Nat. Chem. 2013, 5, 750-756. (e) Prier, C. K.; Rankic, D. A.; MacMillan, D. W. Visible Light Photoredox Catalysis with Transition Metal Complexes: Applications in Organic Synthesis. Chem. Rev. 2013, 113, 5322-5363.

(8) (a) McLean, E. B.; Gauchot, V.; Brunen, S.; Burns, D. J.; Lee, A.L. Dual copper- and photoredox-catalysed $\mathrm{C}\left(\mathrm{sp}^{2}\right)-\mathrm{C}\left(\mathrm{sp}^{3}\right)$ coupling. Chem. Commun. 2019, 55, 4238-4241. (b) Park, G.; Yi, S. Y.; Jung, J.; Cho, E. J.; You, Y. Mechanism and Applications of the Photoredox Catalytic Coupling of Benzyl Bromides. Chem. Eur. J. 2016, 22, 17790 -17799 .

(9) Schweitzer-Chaput, B.; Horwitz, M. A.; Beato, E.; Melchiorre, P. Photochemical generation of radicals from alkyl electrophiles using a nucleophilic organic catalyst. Nat. Chem. 2019, 11, 129-135.

(10) (a) Görner, H. Photoreactions of 2-methyl-5-isopropyl-1,4-benzoquinone. J. Photochem. Photobiol. A: Chem. 2004, 165, 215-222. (b) Görner, $\mathrm{H}$. Photoprocesses of $p$-naphthoquinones and vitamin $\mathrm{K}_{1}$ : effects of alcohols and amines on the reactivity in solution. Photochem. Photobiol. Sci. 2004, 3, 71-78. (c) Görner, H. Photoreduction of nitro1,4-naphthoquinones in solution. J. Photochem. Photobiol. A. 2011, $224,135-140$

(11) Chih, T.; Yeh, S. Y.; Wang, C. M. Evidence and applications for electron transfer between vitamin $\mathrm{K}_{3}$ and oxygen. J. Electroanal. Chem. 2003, 543, 135-142.

(12) (a) For a review on superoxide radical: Hayyan, M.; Hashim, M. A.; Al Nashef, I. M. Superoxide Ion: Generation and Chemical Implications. Chem. Rev. 2016, 116, 3029-3085. (b) Voelker, B. M.; Sedlak, D. L. Iron reduction by photoproduced superoxide in seawater. Mar. Chem. 1995, 50, 93-102. (c) Nunoshiba, T.; Obata, F.; Boss, A. C.; Oikawa, S.; Mori, T.; Kawanishi, S.; Yamamoto, K. Role of Iron and Superoxide for Generation of Hydroxyl Radical, Oxidative DNA Lesions, and Mutagenesis in Escherichia coli. J. Biol. Chem. 1999, 274, 34832 34837. (d) Lueder, U.; Jørgensen, B. B.; Kapplerab, A.; Schmidt, C. Photochemistry of iron in aquatic environments. Environ. Sci.: Processes Impacts, 2020, 22, 12-24.

(13) Lang, K.; Luňák, S. Photocatalytic degradation of 4-chlorophenoxyacetic acid in the presence of an iron complex and hydrogen peroxide. Photochem. Photobiol. Sci. 2002, 1, 588-591.

(14) For a review on intramolecular photoredox reaction of quinones: Ando, Y.; Suzuki, K. Photoredox Reactions of Quinones. Chem. Eur. J. 2018, 24, 15955-15964.

(15) Das, R.; Kapur, M. Palladium-Catalyzed, ortho-Selective C-H Halogenation of Benzyl Nitriles, Aryl Weinreb Amides, and Anilides. J. Org. Chem. 2017, 82, 1114-1126.

(16) Champagne, P. A.; Pomarole, J.; Thérien, M.; Benhassine, Y.; Beaulieu, S.; Legault, C. Y.; Paquin, J. Enabling Nucleophilic Substitution Reactions of Activated Alkyl Fluorides through Hydrogen Bonding. Org. Lett. 2013, 15, 2210-2213.

(17) Gill, A. L.; Frederickson, M.; Cleasby, A.; Woodhead, S. J.; Carr, M. G.; Woodhead, A. J.; Walker, M. T.; Congreve, M. S.; Devine, A.; Tisi, D.; O’Reilly, M; Seavers, L. C. A.; Davis, D. J.; Curry, J.; Anthony, R.; Padova, A.; Murray, C. W.; Carr, R. A. E.; Jhoti, H. Identification of Novel p38 $\alpha$ MAP Kinase Inhibitors Using FragmentBased Lead Generation. J. Med. Chem, 2005, 48, 414-426.

(18) Feng, L.; Lv, K.; Liu, M.; Wang, S.; Zhao, J.; You, X.; Li, S.; Cao, J.; Guo, H. Synthesis and in vitro antibacterial activity of gemifloxacin derivatives containing a substituted benzyloxime moiety. Eur. J. Med. Chem. 2012, 55, 125-136.

(19) Miura, Y.; Issiki, T.; Ushitani, Y.; Teki, Y.; Itoh, K. Synthesis and magnetic behaviour of polyradical: poly(1,3-phenyleneethynylene) with $\pi$-toporegulated pendant stable aminoxyl and imine $N$-oxide-aminoxyl radicals. J. Mater. Chem. 1996, 6, 1745-1750.

(20) Caputo, D. F. J.; Arroniz, C.; Durr, A. B.; Mousseau, J. J.; Stepan, A. F.; Mansfield, S. J.; Anderson, E. A. Synthesis and applications of highly functionalized 1-halo-3-substituted bicyclo[1.1.1]pentanes. Chem. Sci. 2018, 9, 5295-5300. 
(21) Su, J.; Zhang, Y.; Chen, M.; Li, W.; Qin, X.; Xie, Y.; Qin, L.; Huang, S.; Zhang, M. A. Copper Halide Promoted Regioselective Halogenation of Coumarins Using N-Halosuccinimide as Halide Source. Synlett. 2019, 30, 630-634.

(22) She, Z.; Shi, Y.; Huang, Y.; Cheng, Y.; Song, F.; You, J. Versatile palladium-catalyzed $\mathrm{C}-\mathrm{H}$ olefination of (hetero)arenes at room temperature. ChemComm, 2014, 50, 13914-13916.

(23) Bunge, A.; Hamann, H.-J.; McCalmont, E.; Liebscher, J. Enantioselective epoxidation of 2-substituted 1,4-naphthoquinones using gem-dihydroperoxides. Tetrahedron Lett. 2009, 50, 4629-4632.

(24) Bolognesi, M. L.; Lizzi, F.; Perozzo, R.; Brun, R.; Cavalli, A. Synthesis of a small library of 2-phenoxy-1,4-naphthoquinone and 2- phenoxy-1,4-anthraquinone derivatives bearing anti-trypanosomal and anti-leishmanial activity. Bioorg. Med. Chem. Lett. 2008, 18, 22722276.

(25) Smith, R. B.; Canton, C.; Lawrence, N. S.; Livingstone, C.; Davis, J. Molecular anchors-mimicking metabolic processes in thiol analysis. New. J. Chem. 2006, 30, 1718-1724.

(26) Rajendra Prasad, K.; Suresh Babu, K.; Ranga Rao, R.; Suresh, G.; Rekha, K.; Madhusudana Murthy, J.; Usha Rani, P.; Madhusudana Rao, J. Synthesis and insect antifeedant activity of plumbagin derivatives. Med. Chem. Res. 2012, 21, 578-583 